\title{
MONODROMY GROUPS AND LINEARLY POLYMORPHIC FUNCTIONS
}

BY

DENNIS A. HEJHAL

Columbia University New York, N.Y. 10027 USA ( ${ }^{(1)}$

Table of contents

1. Introduction . . . . . . . . . . . . . . . . . . . . . . . . . . . 1

2. Notation . . . . . . . . . . . . . . . . . . . . . . . . . . . . 4

3. General remarks (for arbitrary $n$ ) . . . . . . . . . . . . . . . . . . . . . . 5

4. Further remarks (for $n=2$ ) . . . . . . . . . . . . . . . . . . . . . . . . . 6

5. The hypergeometric equation . . . . . . . . . . . . . . . . . . . . . . . 10

6. Marked monodromy groups and the vector bundle $T Q$. . . . . . . . . . . . . . 14

7. Some known results . . . . . . . . . . . . . . . . . . . . . . . . . 19

8. Statement of the main theorems. . . . . . . . . . . . . . . . . . . . . . . . 20

9. Proofs of the main theorems . . . . . . . . . . . . . . . . . . . . . . . 21

10. Some further results . . . . . . . . . . . . . . . . . . . . . . . . 39

11. Linearly polymorphic functions with ramification . . . . . . . . . . . . . . . . 44

12. Concluding remarks . . . . . . . . . . . . . . . . . . . . . . . . . . 50

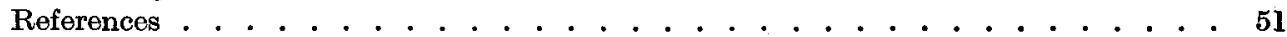

\section{Introduction}

In this paper, we shall be concerned with monodromy groups of second-order linear differential equations on compact Riemann surfaces:

$$
\left.\begin{array}{r}
\frac{d^{2} u}{d x^{2}}+Q_{1}(x, y) \frac{d u}{d x}+Q_{2}(x, y) u=0 \\
P(x, y)=0
\end{array}\right\} .
$$

The coefficients $Q_{k}(x, y)$ are assumed to be rational functions and $P(x, y)$ is assumed to be an irreducible polynomial. There are essentially two main problems in the classical theory of monodromy groups:

(1) This work was done at Harvard University and was supported in part by NSF Grant GP. 38886 and the U.S. Army Research Office (Durham). 
(A) to calculate the group, when the differential equation is given;

(B) to determine all differential equations having a specified monodromy group.

Most of our results will concern problem B for differential equations of the so-called Fuchsian type.

Although there was extensive work on both questions during the last half of the nineteenth century, the over-all state of affairs is still rather primitive (after 70 some years). In part, this may be due to the non-Abelian nature of the problems. Certainly, the classical theory of Abelian integrals and theta functions is much more developed.

A few historical remarks may help to put matters in perspective. First of all, work of the nineteenth century mathematicians on monodromy groups was largely motivated by problerns having to do with uniformization and discontinuous groups. There is in fact a very close connection between these areas. Assume, for instance, that the surface $F: P(x, y)=0$ has genus $g \geqslant 2$. We may therefore represent $F$ in the form $U / \mathcal{G}$, where $U$ is the unit disk and $\mathcal{G}$ is a Fuchsian group. The problem of calculating $\mathcal{G}$ and the universal covering map $\pi: U \rightarrow F$ is essentially equivalent to the notorious problem of choosing correctly the values of $3 g-3$ accessory parameters in a certain rational function $R(x, y)$ and then solving the equation

$$
\frac{d^{2} u}{d x^{2}}+R(x, y) u=0 .
$$

The basic (historical) references here are: Klein [32, 33], Poincaré [47], Riemann [50, pp. 440-444], and Schottky [56, 57]. One may also want to refer to [5, pp. 282-286], [6, pp. 371-372], and [53].

The problem of the accessory parameters has turned out to be quite difficult; in fact, it has not yet received an entirely satisfactory solution. This particular approach to uniformization and discontinuous groups based on differential equations was gradually abandoned after 1900 , when better methods were found.

A second, very important reason for the study of problem $B$ stems from its close connection to the classical Riemann problem (Hilbert problem 21; see also [50, pp. 379-390]). The first attempts at solving the Riemann problem used the zeta-Fuchsian series of Poin. caré [48] and were carried out in the context of differential equations. It turned out, however, that the classical Riemann problem could be solved most directly by using singular integral equations. See, for example, Hilbert [28] and Plemelj [45]. Because of this, zetaFuchsian series (and differential equations) have largely disappeared from modern treatments of the subject: Röhrl [52] gives a useful survey.

There has of course been a revival of interest in various areas of classical function theory: uniformization, Kleinian groups, theta functions, etc. It seems only natural that 
one should take another look at differential equations and their monodromy groups. This is especially true in view of recent results [12] connecting monodromy groups with quasiFuchsian and totally degenerate groups.

Such an undertaking might prove to be of interest, in view of the fact that a careful reading of the work of Poincaré on these topics suggests that a number of his ideas can actually be pushed much further; e.g. the method of continuity.

Schiffer and Hawley [53, p. 209] have in fact raised the possibility of using the monodromy groups of certain second-order equations as a kind of moduli for compact Riemann surfaces. This possibility necessitates looking at problem B in a context in which both the monodromy group and the Riemann surface are allowed to vary. One is very much tempted to apply here a continuity method similar to that of Poincaré.

Generally speaking, the main purpose of this paper is two-fold: (a) to obtain a better understanding of this problem of Schiffer and Hawley; and (b) to clarify certain important aspects of section IV of Poincaré [47]. Parts (a) and (b) are very much interrelated.

Our basic method tends to be quite geometric: we shall actually "look" at fundamental membranes in the spirit of Klein [32, 33, 34, 35] and Poincaré [47].

A brief statement of our results may be helpful at this point. We concern ourselves mainly with locally schlicht, linearly polymorphic (L.P.) functions arising from equations (1.1) of Fuchsian type. We shall prove that:

(1) there exist monodromy groups which look just like ordinary Fuchsian (or Schottky) groups, but which do not correspond to a uniformization. A geometric argument based on the Hopf-Poincaré index theorem for vector fields will be used to study these situations. One important consequence is that the classical accessory parameter problem cannot be solved simply by adjusting the group.

(2) the monodromy mapping $p: T Q \rightarrow m$ from the vector bundle of quadratic differentials to the monodromy space is well-behaved locally, but has some rather unfortunate topological properties globally. For example, it is not even a covering map. As a consequence, the moduli proposed by Schiffer and Hawley can be used only locally.

(3) however, when restricted to the so-called quasi-Fuchsian part of $m$, the monodromy mapping $p$ is a topological covering.

(4) the monodromy space $m$ contains monodromy groups arbitrarily close to the identity. This tends to support the conjecture that $m$ is actually dense in the appropriate ambient space $(N / L F$ below).

(5) the monodromy space $m$ also contains certain rather special groups which have $2 g-2$ out of their $2 g$ generators equal to the identity. 
(6) there seems to exist a rather general method for translating data about ramified L.P. functions into nontrivial information about locally schlicht L.P. functions. This method makes contact with classical algebraic geometry.

As might be expected, the developments we give raise many further questions. We hope to treat some of these questions in subsequent publications. (A list of open problems can be found in section 12.)

Finally, I would like to express my sincere thanks to Professors L. Ahlfors and M. Schiffer for providing me with many helpful remarks and criticisms during the course of this work.

\section{Notation}

The following is a list of various notations and abbreviations used throughout sections 3-12.

D.E. = differential equation

L.F. = linear fractional

L.P. = linearly polymorphic

$\mathrm{NE}=$ non-Euclidean

$\mathrm{QC}=$ quasi-conformal

$\mathrm{WLOG}=$ without loss of generality

iff $=$ if and only if

$\mathbf{C}=$ complex plane

$\mathbf{R}=$ real line

$\hat{\mathbf{C}}=\mathbf{C} \cup\{\infty\}$

$U=$ the open unit disk

$\mathbf{Z}=$ the integers

Cap $(E)=$ the capacity of $E$

$[x]=$ greatest integer function (occasionally)

$\cong$ means isomorphic $A(\mathcal{I})=$ the area of $\mathfrak{I}$ (occasionally)

$\approx$ means approximately equal

$\{f, z\}=$ the Schwarzian derivative of $f(z)$ :

$$
\{f, z\}=\left(\frac{f^{\prime \prime}(z)}{f^{\prime}(z)}\right)^{\prime}-\frac{1}{2}\left(\frac{f^{\prime \prime}(z)}{f^{\prime}(z)}\right)^{2}
$$

$\pi_{1}(F, O)=$ the fundamental group of $F$ with base point $O$

$G L(n, \mathbf{C})=\{M: M$ is an $n \times n$ matrix over $\mathbf{C}, \operatorname{det}(M) \neq 0\}$

$S L(n, \mathbf{C})=\{M: M$ is an $n \times n$ matrix over $\mathbf{C}, \operatorname{det}(\mathbf{M})=1\}$

$s l(n, \mathbf{C})=\{M: M$ is an $n \times n$ matrix over $\mathbf{C}, \operatorname{Tr}(M)=0\}$

$L F(2, \mathbf{C})=$ the set of all linear fractional transformations $((a z+b) /(c z+d))$ over $\mathbf{C}$

$\left[T_{1}, \ldots, T_{n}\right]=$ the group generated by the L.F. transformations $T_{1}, \ldots, T_{n}$

$(X, Y)=X Y X^{-1} Y^{-1}=$ the commutator of $X$ and $Y$ 
The following conventions are also useful:

(a) $E>\phi$ means that the set $E$ is non-void;

(b) the dimensions of all loci are understood to be complex: e.g. $\operatorname{dim}\left(\mathbf{R}^{2 n}\right)=n$;

(c) a function $H\left(z_{1}, \ldots, z_{n}\right)$ is said to be real-analytic iff $\operatorname{Re}(H)$ and $\operatorname{Im}(H)$ can be expanded (locally) as power series in $\operatorname{Re}\left(z_{k}\right), \operatorname{Im}\left(z_{k}\right), 1 \leqslant k \leqslant n$.

\section{General remarks (for arbitrary $n$ )}

Before we get into a more detailed discussion of the case $n=2$, a few basic remarks valid for general $n$th order linear equations may be helpful. We shall use Forsyth [16, pp. 478-525] as a reference.

The local solution space structure of the D.E.

$$
\left.\begin{array}{r}
\frac{d^{n} u}{d x^{n}}+Q_{1}(x, y) \frac{d^{n-1} u}{d x^{n-1}}+Q_{2}(x, y) \frac{d^{n-2} u}{d x^{n-2}}+\ldots+Q_{n}(x, y) u=0 \\
P(x, y)=0
\end{array}\right\}
$$

is most naturally studied by transforming the independent variable from $x$ into $t$, where $t$ is a local coordinate on the surface $F$ defined by $P(x, y)=0$.

Throughout this paper, we restrict ourselves to D.E. of the Fuchsian type, that is, to those having only regular singular points [16, pp. 78, 123]. Note here that the nature of these singular points is determined by use of the local variable $t$.

Let $\mathcal{U}$ denote a column vector of $n$ linearly independent solutions of (3.1). The local situation is quite elementary: as $t$ circles around the regular singular point $t=0, \mathcal{U}$ simply transforms into $M \mathcal{U}$, where $M \in G L(n, \mathbf{C})$.

The global situation is more interesting. Assuming that D.E. (3.1) is of Fuchsian type, we let $E$ denote the (finite) set of regular singular points. We then select any non-exceptional base point $\xi_{0}=\left(x_{0}, y_{0}\right) \in F$ and form a solution vector $U$ near $\xi_{0}$. Now, as we move $U$ along any closed path $\gamma \in \pi_{1}\left(F-E, \xi_{0}\right)$, the vector $\mathcal{U}$ gets transformed into $M(\gamma) \mathcal{U}$, where $M(\gamma) \in G L(n, \mathrm{C})$. It is readily checked that $\gamma \rightarrow M(\gamma)$ defines a homomorphism $M: \pi_{1}\left(F-E, \xi_{0}\right) \rightarrow G L(n, \mathrm{C})$. The image group $\{M(\gamma)\}$ is known as the monodromy group of (3.1).

Because the choices of $\xi_{0}$ and $U$ are not unique, there is a certain ambiguity in the monodromy group. One readily checks, however, that changes in $\xi_{0}$ and $\mathcal{U}$ will affect things by at most inner automorphisms.

It is sometimes rather useful to be able to assume that the monodromy group $\{M(\gamma)\}$ is actually a subgroup of $S L(n, \mathbf{C})$. This can always be achieved by a simple change of variable.

Namely, consider the effect of the substitution: 


$$
v=u \exp \left[\int A(x, y) d x\right]
$$

where $\int A(x, y) d x$ denotes an Abelian integral of the first or third kind on $F$, whose logarithmic singularities are confined to a set $E_{0}$ such that $E \subseteq E_{0}$. It is easily seen that the D.E. for $v$ is still of Fuchsian type, and that its regular singular points are all contained in $E_{0}$. It is also clear that the monodromy groups of the $u$ and $v$ equations are very simply related. One might almost say they are equivalent.

A simple calculation shows that $A(x, y)=(1 / n) Q_{1}(x, y)$ is admissible (for an easily calculated set $E_{0}$ ) and leads to the D.E.

$$
\frac{d^{n} v}{d x^{n}}+R_{2}(x, y) \frac{d^{n-2} v}{d x^{n-2}}+\ldots+R_{n}(x, y) v=0 .
$$

Since the Wronskian for the general equation (3.1) satisfies $W^{\prime}+Q_{1} W=0$, we see that the Wronskian of (3.2) must reduce to a non-zero constant. Notice, however, that the Wronskian determinant satisfies the equation $W[N(\gamma) \vartheta]=W[\vartheta] \operatorname{det}[N(\gamma)]$, where $N(\gamma)$ denotes the monodromy homomorphism for $\vartheta$. It follows therefore that $\operatorname{det}[N(\gamma)]=1$, whence $N(\gamma) \epsilon$ $S L(n, \mathbf{C})$.

Finally, it is frequently useful to think of the solution vector $\mathcal{U}$ as being imbedded in the projective space $P_{n-1}(\mathrm{C})$. It is then quite natural to consider the monodromy group as being a subgroup of the general projective group $P G L(n, \mathrm{C})$. In the case $n=2$, this just corresponds to looking at monodromy groups of linear fractional transformations. However, for $n \geqslant 3$, one encounters some very interesting connections with the classical theory of invariants. See, for example, [16, pp. 174-218], [54, pp. 180-199], and [67]. Not very much work has been done on these higher-dimensional monodromy groups (in the context of differential equations).

Note: It is reasonable to expect, however, that by imposing further restrictions on the equations (3.1) one can obtain a better grip on the corresponding monodromy groups. There are two important examples of this in the literature: (1) the generalized ( $n$th order) hypergeometric equation; and (2) the Picard-Fuchs equation encountered in algebraic geometry. For a discussion of (1), we refer to [27, pp. 549-553], [35], [68], and [92]. See also section 5. For the Picard-Fuchs equation, one may refer to [27, pp. 553-555], [69], [70], [73], [76], and [89].

\section{Further remarks (for $n=2$ )}

We now turn to a more thorough discussion of second-order equations of Fuchsian type: 


$$
\left.\begin{array}{r}
\frac{d^{2} u}{d x^{2}}+Q_{1}(x, y) \frac{d u}{d x}+Q_{2}(x, y) u=0 \\
P(x, y)=0
\end{array}\right\} .
$$

As before, we let $F$ denote the Riemann surface $P(x, y)=0, E$ denote the set of regular singular points, and $U$ denote a solution vector $\left(\begin{array}{l}u_{1} \\ u_{2}\end{array}\right)$ with linearly independent $u_{\alpha}$.

Following Klein [32, 33, 34, 35] and others, we wish to investigate the conformal mapping properties of the multi-valued function $z=u_{1} / u_{2}$. We must therefore consider the projective monodromy group.

Making a substitution $v=u \exp \left[\int A(x, y) d x\right]$ as above will clearly leave $z$ invariant. For this reason, we may assume from the start, whenever helpful, that our D.E. reads

$$
\left.\begin{array}{r}
\frac{d^{2} u}{d x^{2}}+R(x, y) u=0 \\
P(x, y)=0
\end{array}\right\}
$$

A simple calculation with $A(x, y)=\frac{1}{2} Q_{1}(x, y)$ shows that

$$
R(x, y)=Q_{2}(x, y)-\frac{1}{4} Q_{1}(x, y)^{2}-\frac{1}{2} \frac{d Q_{1}}{d x}
$$

where the derivative $d Q_{1} / d x$ is taken with the constraint $P(x, y)=0$. The monodromy group of (4.2) will be a subgroup of $S L(2, \mathrm{C})$.

It is simple to check that $z$ is both locally meromorphic and locally schlicht away from $E$. To ensure reasonable mapping properties of $z$ near the regular singular points, we shall assume that the multi-valued function $z$ is locally meromorphic everywhere on $F$. This will impose certain conditions on $R(x, y)$, as we shall see.

Locally meromorphic or not, the multi-valued function $z$ transforms linear fractionally under the action of $\pi_{1}\left(F-E, \xi_{0}\right)$ :

$$
z \rightarrow L(\gamma) z, \quad L(\gamma) \in L F(2, \mathbf{C}) .
$$

The projective monodromy group $\{L(\gamma)\}$ is defined by the homomorphism $L: \pi_{1}\left(F-E, \xi_{0}\right) \rightarrow$ $L F(2, C)$. Notice, however, that the monodromy near the points of $E$ will betrivial if $z$ is assumed to be locally meromorphic. In this case, one naturally thinks of $L$ as being a homomorphism $\pi_{1}\left(F, \xi_{0}\right) \rightarrow L F(2, \mathrm{C})$.

In the following lemma, we assume that $\xi_{1}=\left(x_{1}, y_{1}\right)$ is a regular singular point and that the local uniformizer satisfies $x-x_{1}=t^{k} \varphi(t), \varphi(0) \neq 0, k \geqslant 1$.

LEMMA 1. A necessary and sufficient condition for the multi-valued function $z$ associated with differential equation (4.2) to be meromorphic at $t=0$ with multiplicity $m \geqslant 1$ is that 


$$
R(x, y) d x^{2}=\left[\frac{k^{2}-m^{2}}{4 t^{2}}+\frac{\alpha}{t}+\sum_{n=0}^{\infty} \alpha_{n} t^{n}\right] d t^{2}
$$

where $\alpha$ satisfies

$$
\left\{\begin{array}{l}
\alpha=\frac{k^{2}-1}{2 k} \frac{\varphi^{\prime}(0)}{\varphi(0)} \quad \text { when } m=1, \\
F\left[\alpha ; \alpha_{0}, \ldots, \alpha_{m-2} ; \varphi(0), \varphi^{\prime}(0), \ldots, \varphi^{(m)}(0)\right]=0 \quad \text { when } m \geqslant 2 .
\end{array}\right.
$$

$F\left[u ; u_{0}, \ldots, u_{m-2} ; v_{0}, \ldots, v_{m}\right]$ is a certain polynomial dependent solely on $k$ and $m$.

Proof. To begin with, we readily make the change of variable $x \rightarrow t$ in (4.2):

$$
\frac{d^{2} u}{d t^{2}}+\left[\frac{1-k}{t}-\frac{\psi^{\prime}(t)}{\psi(t)}\right] \frac{d u}{d t}+R\left(\frac{d x}{d t}\right)^{2} u=0
$$

where $\psi(t)=k \varphi(t)+t \varphi^{\prime}(t)$. Since $t=0$ is a regular singular point, we can automatically write

$$
R(x, y) d x^{2}=\left[\frac{A}{t^{2}}+\frac{\alpha}{t}+\sum_{n=0}^{\infty} \alpha_{n} t^{n}\right] d t^{2}
$$

Observe, however, that the functions $u_{1}=z(d x / d z)^{1 / 2}, u_{2}=(d x / d z)^{1 / 2}$ are solutions of (4.2). It follows that $z$ is meromorphic at $t=0$ with multiplicity $m$ iff the D.E. (4.4) has indicial equation $R^{2}-k R+\frac{1}{4}\left(k^{2}-m^{2}\right)=0$ and only non-logarithmic solutions at $t=0$.

The problem is now reduced to that of writing down the condition for the D.E.'(4.4) with $A=\frac{1}{4}\left(k^{2}-m^{2}\right)$ to have only non-logarithmic solutions [16, p. 106]. This condition can be found by straightforward substitution of $u=\Sigma_{n=0}^{\infty} c_{n} t^{R+n}$ and careful study of the resulting recurrence relation:

$$
c_{n}\left[(R+n)^{2}-k(R+n)+\frac{1}{4}\left(k^{2}-m^{2}\right)\right]=\sum_{\mu+v=n-1}(R+\mu) c_{\mu} \psi_{v}-\alpha c_{n-1}-\sum_{\mu+v=n-2} c_{\mu} \alpha_{v}
$$

The Taylor coefficients of $\psi^{\prime}(t) / \psi(t)$ are denoted here by $\psi_{v}$. The critical case is easily seen to be $R=\frac{1}{2}(k-m)$ and $n=m$.

Remark. We omit the trivial modifications called for when $x_{1}=\infty$. (One simply takes $k<0$ in the above.)

Suppose next that we are given a nonconstant locally meromorphic function $w$ on $F$ which transforms linear fractionally under the action of $\pi_{1}\left(F, \xi_{0}\right)$.

LEMMA 2. Every such multi-valued function $w$ arises from some differential equation (4.2) of Fuchsian type.

Proof. The Schwarzian derivative $\{w, x\}$ is immediately seen to be a single-valued meromorphic function on $F$; the local behavior is easily studied using the identity 


$$
\{w, x\} d x^{2}+\{x, t\} d t^{2}=\{w, t\} d t^{2} .
$$

We shall therefore write $\{w, x\}=2 R(x, y)$ and consider the D.E.

$$
\frac{d^{2} u}{d x^{2}}+R(x, y) u=0 \text {. }
$$

This D.E. is easily seen to be of Fuchsian type via (4.4). Direct computation proves that the associated function $z=u_{1} / u_{2}$ satisfies

$$
\{z, x\}=2 R(x, y) .
$$

It follows that $w=T z$ for some $T \in L F^{\prime}(2, \mathbf{C})$, which proves the lemma.

As the proof clearly shows, there is a natural correspondence between the differential equations $\{w, x\}=2 R(x, y)$ and $u^{\prime \prime}+R(x, y) u=0$. This is a well-known classical fact [16, p. 495], which will be used frequently in all that follows.

A locally meromorphic function which transforms linear fractionally under $\pi_{1}\left(F, \xi_{0}\right)$ is called linearly polymorphic.

Linearly polymorphic functions which have $m=1$ at every point of $F$, that is, which are locally schlicht, play a very important role in modern uniformization and Teichmüller theory. See, for example, Bers [12]. We shall work mainly with such functions in this paper.

The connection with uniformization can be seen quite easily. Assume, for example, that $\pi: U \rightarrow F$ is the universal covering map for $F$. The multivalued inverse function $w=\pi^{-1}(\xi)$ will then be a locally schlicht L.P. function. The associated monodromy group will be a Fuchsian group $\mathcal{G}: F=U / \mathcal{G}$.

Similarly, if $D \subseteq \hat{\mathrm{C}}$ denotes the Schottky covering surface of $F$, the covering map $\psi: D \rightarrow F$ will give rise to a locally schlicht L.P. function $w=\psi^{-1}(\xi)$ whose monodromy group is a Schottky group $S: F=D / S$. See [2, pp. 239-243], [5, pp. 484-495], and [23].

We remark that there are natural examples of locally schlicht L.P. functions which (apparently) have nothing to do with uniformization. One such example can be found in [53, pp. 203-204] and also in [57, pp. 265-272]. This particular example is important because it requires only an Abelian integral of the third kind.

Let $R_{0}(x, y)$ be the rational function corresponding to any one of the above three examples. Lemmas 1 and 2 show that the most general locally schlicht L.P. function on $F$ can be obtained by solving the Schwarzian D.E.

$$
\{w, x\}=2\left[R_{0}(x, y)+Q(x, y)\right],
$$

where $Q(x, y) d x^{2}$ is any regular quadratic differential on $F$. If we let $\left\{Q_{1}, \ldots, Q_{N}\right\}$ be a basis for such quadratic differentials, 
then we clearly obtain

$$
N= \begin{cases}3 g-3, & g \geqslant 2 \\ 1, & g=1, \\ 0, & g=0\end{cases}
$$

$$
\{w, x\}=2\left[R_{0}(x, y)+\sum_{j=1}^{N} \lambda_{j} Q_{j}(x, y)\right]
$$

The complex constants $\lambda_{j}$ are precisely the accessory parameters mentioned in section 1 . The corresponding linear D.E. is

$$
\frac{d^{2} u}{d x^{2}}+\left[R_{0}(x, y)+\sum_{j=1}^{N} \lambda_{j} Q_{j}(x, y)\right] u=0
$$

One naturally wonders what happens to $w$ and its monodromy group as the parameters $\lambda_{1}, \ldots, \lambda_{N}$ vary. A complete answer to this question has proved quite elusive. See also [24] and $[25]$.

Suppose, finally, that $F$ has genus $g \geqslant 2$. We let $(U, \pi)$ be the universal covering surface of $F$ and let $\mathcal{G}$ denote the automorphic group: $F=U / G$. Let us fix $t_{0} \in U$ so that $\pi\left(t_{0}\right)=$ $\xi_{0}$. The general theory of covering surfaces [2, pp. 34-39] then yields a natural isomorphism $\pi_{1}\left(F, \xi_{0}\right) \cong \mathcal{G}$.

It follows that, when lifted to $(U, \pi)$, linearly polymorphic functions are simply meromorphic functions $z(t)$ on $U$ which satisfy a transformation law

$$
z(L t)=\chi(L) z(t), \quad \chi(L) \in L F(2, \mathbf{C}),
$$

for $L \in \mathcal{G}$. The mapping $L \rightarrow \chi(L)$ is seen to be a homomorphism $\mathcal{G} \rightarrow L F(2, \mathrm{C})$.

The function $q(t)=\{z, t\}$ satisfies the equation $q(L t) L^{\prime}(t)^{2}=q(t)$ for $L \in \mathcal{G} . q(t)$ can therefore be thought of as a quadratic differential on $F=U / G$. It is easily seen that locally schlicht L.P. functions correspond to regular quadratic differentials $q(t)$.

Throughout the following sections, we shall work on either $F$ or $(U, \pi)$ as dictated by convenience. That is: on $F$ in section 5 , and on $(U, \pi)$ in sections 6-12.

\section{The hypergeometric equation}

In an ideal situation, one would be able to calculate the monodromy group of any linear D.E. by some sort of explicit formula. (We recall problem A.) Unfortunately, the actual state of affairs is far from ideal, and we can carry out the explicit computation of monodromy groups for only a few special cases.

The most important such case is that of the classical hypergeometric equation. The necessary formulas for calculating its monodromy group can be found in [7, pp. 93-95], 
[16, pp. 135-150], and [35, pp. 53-57]. Comparatively speaking, the simplicity of these formulas stems from the fact that the hypergeometric equation involves only the Riemann sphere $\hat{\mathbf{C}}$ and three regular singular points.

In later sections of this paper, the situation becomes much more complicated and we will be forced to side-step problem A entirely. This will be done by working strictly with L.P. functions and their monodromy groups, letting the associated differential equations (such as (4.6)) fall where they may.

As a matter of principle, then, we should try to calculate at least one example fairly explicitly (including both the group and the D.E.). To do so, we naturally use the hypergeometric equation.

Our basic reference for the hypergeometric equation will be the excellent book by Klein [35].

We begin by recalling the Riemann-Papperitz equation [35, pp. 26, 116]:

$$
\begin{aligned}
\frac{d^{2} u}{d x^{2}} & +\left\{\frac{1-\alpha_{1}-\alpha_{2}}{x-a}+\frac{1-\beta_{1}-\beta_{2}}{x-b}+\frac{1-\gamma_{1}-\gamma_{2}}{x-c}\right\} \frac{d u}{d x} \\
& +\left\{\frac{\alpha_{1} \alpha_{2}(a-b)(a-c)}{x-a}+\frac{\beta_{1} \beta_{2}(b-a)(b-c)}{x-b}+\frac{\gamma_{1} \gamma_{2}(c-a)(c-b)}{x-c}\right\} \frac{u}{(x-a)(x-b)(x-c)}=0,
\end{aligned}
$$

where $\alpha_{1}+\alpha_{2}+\beta_{1}+\beta_{2}+\gamma_{1}+\gamma_{2}=1$. This is the general second order equation of Fuchsian type on $\hat{\mathbf{C}}$ with three regular singular points $a, b, c$. The roots of the respective indicial equations are $\alpha_{1}, \alpha_{2} ; \beta_{1}, \beta_{2} ;$ and $\gamma_{1}, \gamma_{2}$. For simplicity, we assume that none of the exponent differences $\alpha_{1}-\alpha_{2}, \beta_{1}-\beta_{2}, \gamma_{1}-\gamma_{2}$ is an integer. The quotient function $z=u_{1}(x) / u_{2}(x)$ is readily seen to be locally schlicht for $x \neq a, b, c$ (since the Wronskian is easily calculated). Near $x=a, b, c$, the function $z(x)$ obviously behaves like $(x-a)^{\alpha_{1}-\alpha_{3}},(x-b)^{\beta_{1}-\beta_{z}},(x-c)^{\gamma_{1}-\gamma_{z}}$.

To obtain the classical hypergeometric equation requires two simple normalizations. We first apply an auxiliary L.F. mapping to assume that $(a, b, c)=(0, \infty, 1)$. This yields [35, pp. 25-26]:

$$
\frac{d^{2} u}{d x^{2}}+\left\{\frac{1-\alpha_{1}-\alpha_{2}}{x}+\frac{1-\gamma_{1}-\gamma_{2}}{x-1}\right\} \frac{d u}{d x}+\left\{\frac{\alpha_{1} \alpha_{2}}{x^{2}}+\frac{\gamma_{1} \gamma_{2}}{(x-1)^{2}}+\frac{\beta_{1} \beta_{2}-\alpha_{1} \alpha_{2}-\gamma_{1} \gamma_{2}}{x(x-1)}\right\} u=0 .
$$

We then set $u=x^{\alpha_{1}}(1-x)^{\gamma_{1}} v$ to obtain the classical form of the hypergeometric equation [35, p. 4]:

$$
x(\mathrm{l}-x) v^{\prime \prime}+[c-(a+b+1) x] v^{\prime}-a b v=0 .
$$

This transformation can be indicated more precisely using the Riemann $P$-function [35, pp. 119-120]: 


$$
\left.\begin{array}{l}
u=P\left(\begin{array}{llll}
0 & \infty & 1 \\
\alpha_{1} & \beta_{1} & \gamma_{1} ; & x \\
\alpha_{2} & \beta_{2} & \gamma_{2}
\end{array}\right), \\
\left.v=P\left(\begin{array}{lllll}
0 & \infty & 1 & & \\
0 & \alpha_{1}+\beta_{1}+\gamma_{1} & 0 & ; & x \\
\alpha_{2}-\alpha_{1} & \alpha_{1}+\beta_{2}+\gamma_{1} & \gamma_{2}-\gamma_{1}
\end{array}\right)=P\left(\begin{array}{lllll}
0 & \infty & 1 & \\
0 & a & 0 & ; \\
1-c & b & c-a-b & x
\end{array}\right\}\right)
\end{array}\right\}
$$

The roots of the indicial equations are very clearly displayed in this way. We might also observe that $z(x)=v_{1}(x) / v_{2}(x)$.

Suppose next that $F$ is any compact Riemann surface whose branch points are situated over $0, \infty, 1$. The function $z(x)$ clearly lifts to $F$. To ensure that $z(x)$ is an L.P. function on $F$, we must impose certain obvious conditions at the branch points (expressed in terms of the local coordinate $t$ ):

$$
\left.\begin{array}{rl}
x=t^{k} & \Rightarrow k\left(\alpha_{1}-\alpha_{2}\right) \in \mathbf{Z} \\
x=t^{-k} & \Rightarrow k\left(\beta_{1}-\beta_{2}\right) \in \mathbf{Z} \\
x-1=t^{k} & \Rightarrow k\left(\gamma_{1}-\gamma_{2}\right) \in \mathbf{Z}
\end{array}\right\} .
$$

The conditions needed to guarantee that $z$ is locally schlicht everywhere on $F$ are similar:

$$
\left.\begin{array}{rl}
x=t^{k} & \Rightarrow k\left(\alpha_{1}-\alpha_{2}\right)= \pm 1 \\
x=t^{k} & \Rightarrow k\left(\beta_{1}-\beta_{2}\right)= \pm 1 \\
x-1=t^{k} & \Rightarrow k\left(\gamma_{1}-\gamma_{2}\right)= \pm 1
\end{array}\right\} .
$$

Example. Consider the surface $F: y^{5}=x(x-1)$. This surface has three branch points $0, \infty, 1$ with the respective local coordinates $x=t^{5}, x=t^{-5}, x-1=t^{5}$. By the Riemann-Hurwitz formula $w=2 n+2 g-2$, the surface $F$ has genus $g=2$.

We are interested in the L.P. functions $z(x)$ on $F$ obtained by lifting equation (5.3). There are two cases of exceptional interest:

(A) $(\Delta \alpha, \Delta \beta, \Delta \gamma)=(2 / 5,2 / 5,2 / 5)$;

(B) $(\Delta \alpha, \Delta \beta, \Delta \gamma)=(4 / 5,1 / 5,1 / 5)$.

According to [35, pp. 261-262], the monodromy group in these two cases will be finite. This should prove interesting, since the finite groups of L.F. transformations are completely tabulated: [15, p. 133], [31, p. 115], [35, p. 257].

We let the corresponding L.P. functions be $z_{1}$ and $z_{2}$, respectively. The behavior of $z_{1}$ and $z_{2}$ at the branch points $0, \infty, 1$ is (up to L.F. mapping): 


\begin{tabular}{lll}
0 & $\infty$ & 1 \\
\hline$z_{1} \approx t^{2}$ & $z_{1} \approx t^{-2}$ & $z_{1} \approx t^{2}$ \\
$z_{2} \approx t^{4}$ & $z_{2} \approx t^{-1}$ & $z_{2} \approx t$
\end{tabular}

At non-branch points, $z_{1}$ and $z_{2}$ are locally schlicht. We thus see that $z_{1}$ has multiplicity 2 at each branch point, but is locally schlicht elsewhere. Similarly, $z_{2}$ has multiplicity 4 at the branch point over $x=0$, but is locally schlicht everywhere else.

By virtue of [35, pp. 261-262], the monodromy groups $m_{1}$ and $m_{2}$ of $z_{1}$ and $z_{2}$ must be subgroups of icosahedral groups $m_{A}$ and $m_{B}$. Observe here that $z_{1}$ and $z_{2}$ are being considered on $F$, not on $\hat{\mathbf{C}}$. Furthermore, as is well-known, $m_{A} \cong m_{B} \cong$ alternating group $A_{5}$ of order 60 [31, pp. 16-19].

We want to calculate $m_{1}$ and $m_{2}$. To do so, let $F_{0}$ be the portion of $F$ over $\hat{\mathbf{C}}-\{0,1, \infty\}=T . F_{0}$ is then a topological covering surface of $T$ and the usual properties now apply $[2,29]$. We let $p: F_{0} \rightarrow T$ be the obvious projection map and write $p\left(\xi_{0}\right)=x_{0}$. It is not difficult to check that $p\left[\pi_{1}\left(F_{0}, \xi_{0}\right)\right]=N$ is a normal subgroup of $\pi_{1}\left(T, x_{0}\right)$ and that the quotient group $\pi_{1}\left(T, x_{0}\right) / N$ is a cyclic group of order 5 .

Consider, for example, the L.P. function $z_{1}$ on $T$. There is certainly a natural homomorphism $\chi: \pi_{1}\left(T, x_{0}\right) \rightarrow m_{A}$ corresponding to the monodromy group of $z_{1}$ (on $\hat{\mathbf{C}}$ ). The mapping $\chi$ is onto [35, pp. 261-262]. We must calculate $m_{1}=\chi(N)$.

First of all, we claim that the order $\left|m_{1}\right| \geqslant 12$. This is immediately seen by applying $\chi$ to a coset decomposition $\pi_{1}(T)=\sum_{i=1}^{5} N \gamma_{i}$.

Secondly, we claim that $m_{1}$ is a normal subgroup of $m_{A}$. This can be checked quite readily, by using the fact that $N$ is a normal subgroup of $\pi_{1}(T)$ and that $\chi$ is onto.

Now for the trick! Since $A_{5} \cong m_{A}$ is a simple group [31, p. 18], we conclude that $m_{1}=$ $m_{A}$. A similar argument shows that $m_{2}=m_{B}$. We have thus proved the curious fact that $m_{1}$ and $m_{2}$ are both icosahedral groups:

$$
m_{1} \cong m_{2} \cong A_{5}
$$

The actual monodromy coefficients can now be calculated by labelling the sheets of $F$, determining a canonical homotopy basis, and substituting into the formulas of [7, pp. 93-95]. The detailed computations are somewhat tedious and will therefore be omitted. In addition to various trigonometric expressions, gamma factors such as

$$
\frac{\Gamma\left(\frac{2}{5}\right)^{2}}{\Gamma\left(\frac{1}{10}\right) \Gamma\left(\frac{7}{10}\right)}
$$

will appear.

The L.P. functions $z_{1}$ and $z_{2}$ have points of ramification (branch points), as noted above. To obtain an example of a locally schlicht L.P. function, it will suffice to take 
$(\Delta \alpha, \Delta \beta, \Delta \gamma)=(1 / 5,1 / 5,1 / 5)$. The monodromy group in this case will necessarily be infinite (see Theorem $\mathrm{C}$ in section 7 ) and will involve gamma factors such as

$$
\frac{\Gamma\left(\frac{4}{5}\right)^{2}}{\Gamma\left(\frac{1}{5}\right) \Gamma\left(\frac{2}{5}\right)} \text {. }
$$

Remark 1. The idea of "lifting" monodromy groups is a trick which we shall use repeatedly in later sections. It appears here in its simplest form. See also [47, section XVI].

Remark 2. The gamma factors cited above give some inkling into the "level of transcendence" of arbitrary monodromy groups. See [37, p. 31].

\section{Marked monodromy groups and the vector bundle $T Q$}

We now turn our attention more towards problem B and, in particular, toward the problem of Schiffer and Hawley mentioned in section 1. We shall be concerned only with locally schlicht L.P. functions (until we reach section 11). According to Lemma 2 of section 4, it makes little difference whether we study such L.P. functions directly or do so using the associated D.E.

$$
\begin{array}{ll}
\{z, x\}=2 R(x, y), & \frac{d^{2} u}{d x^{2}}+R(x, y) u=0 \\
\{z, t\}=2 q(t), \quad \frac{d^{2} u}{d t^{2}}+q(t) u=0 .
\end{array}
$$

We remark that the above two Schwarzian D.E. are related quite simply via

$$
\{z, x\} d x^{2}+\{x, t\} d t^{2}=\{z, t\} d t^{2} .
$$

We need to investigate the situation in which both the quadratic differential and the surface can vary. For this reason, it seems most convenient to work with marked Riemann surfaces and Teichmüller space $T_{g}$.

We shall therefore assume a certain familiarity with modern Teichmüller theory. A very good survey can be found in [13].

We recall a few important facts in this connection. First of all, we had better assume that:

the genus $g \geqslant 2$ is fixed once and for all.

Consider then any marked surface $\left[F,\left\{\mathcal{A}_{k}, \mathcal{B}_{k}\right\}\right]$. Thus $\left\{\mathcal{A}_{k}, \mathcal{B}_{k}\right\}_{k=1}^{g}$ is a canonical dissection for $F$. The loops will have a common base point $O$, the appropriate intersection numbers, and will satisfy the commutator relation 


$$
\left(\mathcal{A}_{1}, \mathcal{B}_{1}\right)\left(A_{2}, B_{2}\right) \ldots\left(\mathcal{A}_{g}, B_{\theta}\right)=I
$$

in $\pi_{1}(F, O)$.

Suppose next that $(U, \pi)$ is a universal covering surface for $F$. We choose $t_{0} \in U$ so that $\pi\left(t_{0}\right)=0$. As was already noted in section 4, there will then exist a natural isomorphism between $\pi_{1}(F, O)$ and the automorphic group $\mathcal{G}$, where $F=U / \mathcal{G}$. (This isomorphism depends upon $t_{0}$.) Because $\pi_{1}(F, O) \cong \mathcal{G}$, the marking on $F$ induces a marking on $G$. There will thus be a distinguished set of generators for $\mathcal{G}$ :

$$
\mathcal{G}=\left[A_{1}, \ldots, A_{g} ; B_{1}, \ldots, B_{g}\right]
$$

Observe too that

$$
\left(A_{1}, B_{1}\right)\left(A_{2}, B_{2}\right) \ldots\left(A_{g}, B_{g}\right)=I .
$$

Equations (6.4) and (6.5) display $\mathcal{G}$ as a marked Fuchsian group.

This representation for $\mathcal{G}$ has two obvious sources of ambiguity, since neither $(U, \pi)$ nor the choice of $t_{0}$ is unique. It is simple to see that this ambiguity affects things by at most an inner automorphism.

To avoid these ambiguities, we use the fact that one can construct a single-valued mapping $\tau \rightarrow \mathcal{G}_{\tau}$ carrying $T_{g}$ into the space of marked Fuchsian groups. This is done by properly normalizing the fixpoints of $A_{1}$ and $B_{1}$. Note here that the fixpoints of $A_{1}$ and $B_{1}$ are mutually distinct, since otherwise $\left(A_{1}, B_{1}\right)$ would be parabolic. We shall write:

$$
\mathcal{G}_{\tau}=\left[A_{1}(\tau), \ldots, A_{g}(\tau) ; B_{1}(\tau), \ldots, B_{g}(\tau)\right] .
$$

This mapping can be used to prove that $T_{g} \cong \mathbf{R}^{6-6}$. See, for example, [8], [9, pp. 112-118], and [63].

Suppose now that we are given a locally schlicht L.P. function on the marked Riemann surface $F$. By representing $F$ as $U / \mathcal{G}_{\tau}$, we obtain a meromorphic function $z(t)$ on $U$ which transforms according to

$$
z(L t)=\tilde{L} z(t), \quad L \in \mathcal{G}_{t}, \quad L \in L F(2, \mathbf{C})
$$

The mapping $L \rightarrow L$ is simply the monodromy homomorphism. We define the marked monodromy group $m[z]$ via

$$
m[z]=\left[\tilde{A}_{1}(\tau), \ldots, \tilde{A}_{g}(\tau) ; \tilde{B}_{1}(\tau), \ldots, \tilde{B}_{g}(\tau)\right] .
$$

The marked monodromy group is uniquely determined by the initial element of $z$ and by $F$. (It is not taken modulo inner automorphisms.) It is also clear that

$$
\left(\tilde{A}_{1}, \tilde{B}_{1}\right)\left(\tilde{A}_{2}, \tilde{B}_{2}\right) \ldots\left(\tilde{A}_{g}, \tilde{B}_{g}\right)=I \text {, }
$$

and that $m[z]$ can be regarded as an element of $L F(2, \mathbf{C})^{20}$. 
Let $R$ be the conglomeration of all marked monodromy groups $m[z]$ over all possible $z$ and $F$. Define:

$$
\begin{aligned}
N & =\left\{\left(X_{1}, \ldots, X_{o} ; Y_{1}, \ldots, Y_{o}\right) \in L F(2, \mathrm{C})^{2 o}:\left(X_{1}, Y_{1}\right) \ldots\left(X_{g}, Y_{g}\right)=I\right\} \\
N_{0} & =\left\{\left(X_{\alpha} ; Y_{\beta}\right) \in N: T X_{\alpha}=X_{\alpha} T, T Y_{\beta}=Y_{\beta} T \text { for } T \in s l(2, \mathrm{C}) \Leftrightarrow T=0\right\} ; \\
N_{1} & =\left\{\left(X_{\alpha} ; Y_{\beta}\right) \in N_{0}: \text { the } X_{\alpha} \text { and } Y_{\beta} \text { have no common fixpoint }\right\} .
\end{aligned}
$$

The term $s l(2, \mathbf{C})$ is defined in section 2 and the subscripts $\alpha, \beta$ are taken over all $1 \leqslant \alpha \leqslant g$, $1 \leqslant \beta \leqslant g$. Using Theorems A-C of section 7, we easily check that $R \subseteq N_{1}$. See also [5, pp. 55-64]. It follows that

$$
m=\frac{R}{L F(2, \mathrm{C})} \subseteq \frac{N_{1}}{L F(2, \mathrm{C})} \subseteq \frac{N_{0}}{L F(2, \mathrm{C})} \subseteq \frac{N}{L F(2, \mathrm{C})} \subseteq \frac{L F(2, \mathrm{C})^{2 g}}{L F(2, \mathrm{C})} \mathrm{a}
$$

The quotient spaces are taken here with respect to the equivalence relation defined by

$$
\left(X_{\alpha} ; Y_{\beta}\right) \sim\left(W X_{\alpha} W^{-1} ; W Y_{\beta} W^{-1}\right) \text { for } W \in L F^{\prime}(2, \mathbf{C}),
$$

and are assumed to carry the obvious topologies.

Gunning [19, 20] has studied some of the properties of the last three terms in (6.10). In so doing, he has carefully analyzed the rank of the Jacobian matrix of the commutator function $\left(X_{1}, Y_{1}\right) \ldots\left(X_{\theta}, Y_{g}\right)$ along $N$. It turns out that: (i) the subvariety $N$ has definite singularities; (ii) $N_{0}$ is open and dense in $N$; (iii) the subvariety $N_{0}$ is a non-singular locus of dimension $6 g-3$.

Discussion of the quotient spaces is somewhat more complicated.

Fact: the quotient space $N_{0} / L F$ is not Hausdorff.

Proof. For simplicity, take $g=2$. We define:

$$
\begin{array}{ll}
A_{1}: \frac{w-1}{w}=\lambda \frac{z-1}{z}, & A_{2}: \frac{w-2}{w}=\mu \frac{z-2}{z} ; \\
\bar{A}_{1}: \frac{w-10}{w-3}=\lambda \frac{z-10}{z-3}, & \bar{A}_{2}: \frac{w-10}{w-4}=\mu \frac{z-10}{z-4} ; \\
H_{n}(z)=\frac{10 n z+1}{n z+1}, & H_{n}\left(a_{n}\right)=3, H_{n}\left(b_{n}\right)=4, a_{n}=\frac{2}{7 n}, b_{n}=\frac{1}{2 n} ; \\
A_{1}^{(n)}: \frac{w-1}{w-a_{n}}=\lambda \frac{z-1}{z-a_{n}}, & A_{2}^{(n)}: \frac{w-2}{w-b_{n}}=\mu \frac{z-2}{z-b_{n}} ; \\
1<\lambda<\infty, & 1<\mu<\infty .
\end{array}
$$

An elementary calculation shows that $A_{1}^{(n)} \rightarrow A_{1}, A_{2}^{(n)} \rightarrow A_{2}$ in $L F(2, \mathbf{C})$. Observe, however, that: 


$$
\begin{aligned}
& H_{n} A_{1}^{(n)} H_{n}^{-1}: \frac{w-H_{n}(1)}{w-3}=\lambda \frac{z-H_{n}(1)}{z-3} ; \\
& H_{n} A_{2}^{(n)} H_{n}^{-1}: \frac{w-H_{n}(2)}{w-4}=\lambda \frac{z-H_{n}(2)}{z-4},
\end{aligned}
$$

and that $\lim _{n \rightarrow \infty} H_{n}(1)=\lim _{n \rightarrow \infty} H_{n}(2)=10$. Therefore, $H_{n} A_{1}^{(n)} H_{n}^{-1} \rightarrow A_{1}, H_{n} A_{2}^{(n)} H_{n}^{-1} \rightarrow A_{2}$ in $L F(2, \mathbf{C})$.

The points $\left(A_{1}, A_{2} ; A_{1}, A_{2}\right),\left(\bar{A}_{1}, \bar{A}_{2} ; \bar{A}_{1}, \bar{A}_{2}\right),\left(A_{1}^{(n)}, A_{2}^{(n)} ; A_{1}^{(n)}, A_{2}^{(n)}\right)$ are easily seen to lie in $N_{0}$ (by studying the fixpoints). A similar analysis shows that $\left(A_{1}, A_{2} ; A_{1}, A_{2}\right) \neq$ $\left(\bar{A}_{1}, \bar{A}_{2} ; \bar{A}_{1}, \bar{A}_{2}\right) \bmod L F(2, \mathbf{C})$.

The projection map $P: N_{0} \rightarrow N_{0} / L F$ is continuous (by definition). We can therefore apply $P$ to the convergent sequences $\left(A_{1}^{(n)}, A_{2}^{(n)} ; A_{1}^{(n)}, A_{2}^{(n)}\right)$ and $H_{n}\left(A_{1}^{(n)}, A_{2}^{(n)} ; A_{1}^{(n)}, A_{2}^{(n)}\right)$ $\cdot H_{n}^{-1}$ to deduce that $N_{0} / L F$ is non-Hausdorff. See [30, pp. 67, 98].

Because of this fact, we have introduced the space $N_{1}$ above. The following properties are rather easily checked using [30] and simple compactness arguments for L.F. maps: (a) $N_{1}$ is a dense, open subset of $N_{0}$; (b) the projection $P: N_{1} \rightarrow N_{1} / L F$ is both open and continuous; (c) the quotient space $N_{1} / L F$ is Hausdorff; (d) $N_{1} / L F$ is locally compact, separable, and metrizable. But, most importantly, by adapting methods found in [19, pp. 186-196] and $[20, \mathrm{pp} .50-53]$, one can prove that $N_{1} / L F$ is actually a complex analytic manifold of dimension $6 g-6$ and that the projection $P$ defines an analytic fibre bundle. We remark that similar methods were already used by Teichmüller in [63, pp. 5-16].

We now return to monodromy groups. We want to define the monodromy mapping $p: T Q \rightarrow m$. Informally, the construction is as follows. Suppose that $\tau \in T_{o}$ is any marked surface and that $q(t) d t^{2}$ is any regular quadratic differential on $U / \mathcal{G}_{\tau}$. The Schwarzian D.E. $\{z, t\}=2 q(t)$ does not have a unique solution; the corresponding marked monodromy group will therefore be determined only up to an inner automorphism. The monodromy mapping $p$ is accordingly defined by $p:(\tau, q) \rightarrow m[z] \bmod L F(2, \mathbf{C})$.

To make matters more precise, we must carefully define the space whose elements are the $(\tau, q)$. This is done in three steps: (I) to each $\tau \in T_{0}$, we associate the complex vector space $Q[\tau]$ of regular quadratic differentials on $U / \mathcal{G}_{\tau}: \operatorname{dim} Q[\tau]=3 g-3$; (II) we observe that locally the spaces $Q[\tau]$ can be supplied with a basis $\left\{q_{k}(t ; \tau)\right\}_{k=1}^{3 g-8}$ which varies real-analytically with $\tau$; and (III) we paste the local situations together to construct a real-analytic vector bundle $T Q$ over $T_{g} . T Q$ is simply the vector bundle of regular quadratic differentials over $T_{g}$.

To avoid getting side-tracked, we shall give additional information about steps (II) and (III) in an appendix.

2-752905 Acta mathematica 135. Imprimé le 19 Déceebre 1975 
The monodromy mapping is now defined rigorously as follows:

$$
\left.\begin{array}{l}
p: T Q \rightarrow m, \\
p:(\tau, q) \rightarrow m[z] \bmod L F(2, \mathrm{C}) .
\end{array}\right\}
$$

Our main goal is to understand the topological properties of the mapping $p: T Q \rightarrow m$ and the imbedding $m \subseteq N_{1} / L F$. The motivation for this comes from [5, pp. 272-379], [47], [48], and [53].

The vector bundle $T Q$ obviously has dimension $6 g-6$ and one would certainly expect $m$ to have the same dimension. The simplest possibility is for $p$ to be a homeomorphism. Some remarks to this effect can, in fact, be found in Poincaré [47, section IV]. His remarks are not very rigorous, however, and several authors have criticized them [5, pp. 310-311, 335-337], [27, p. 518]. Getting closer to the bottom of this question is our most immediate aim.

Appendix. It remains to fill in certain details in the construction of $T Q$. Assume, to begin with, that $T_{g}$ carries the usual Teichmüller topology and that the normalized Fuchsian groups $\mathcal{G}_{\tau}$ are identified with points of $\mathbf{R}^{8 g-6}$ (in an obvious way). As shown by Bers [9, pp. 112-118] and Teichmüller [63], the correspondence $\tau \rightarrow \mathcal{G}_{\tau}$ is actually a homeomorphism. We supply $T_{s}$ with an obvious real-analytic structure $\mathcal{W}$ by means of this homeomorphism.

We shall indicate how to establish step (II) in terms of the structure $W$. This can be done in two ways, both of which involve Poincaré series $\Sigma H(T z) T^{\prime}(z)^{2}$.

The first way uses a well-known completness result $[10,11,46,48]$, applicable when $H(t)$ is a polynomial. One simply expresses the basis $\left\{q_{k}\left(t ; \tau_{0}\right)\right\}_{k=1}^{30-3}$ in terms of such Poincaré series and then perturbs the point $\tau \leftrightarrow \mathcal{G}_{\tau}$ (leaving the associated polynomials $H_{k}(t)$ fixed). For $\tau \approx \tau_{0}$, this process will clearly yield the required sort of basis $\left\{q_{k}(t ; \tau)\right\}_{k=1}^{3 g-3}$.

The second method uses a local form of simultaneous uniformization. Poincaré series (with $H$ rational) can be used to represent the points $\tau \approx \tau_{0}$ as algebraic Riemann surfaces $P(x, y ; \tau)=0$ which are uniformized by functions $x=\xi(t ; \tau), y=\eta(t ; \tau)$ such that:

(a) $P(x, y ; \tau)$ is an irreducible polynomial in $(x, y)$ whose coefficients depend realanalytically on $\tau$;

(b) the functions $\xi(t ; \tau)$ and $\eta(t ; \tau)$ are meromorphic in $t(t \in U)$ and real-analytic in $\tau$;

(c) $t \rightarrow(\xi(t ; \tau), \eta(t ; \tau))$ is the normalized universal covering map for $\tau$.

The basis $\left\{q_{k}(t ; \tau)\right\}$ is then obtained by working directly on the algebraic Riemann surface $P(x, y ; \tau)=0$ using (a)-(c). Further details about this method can be found in [5, pp. 268270] and [23]. 
Step (III) is now established by using the basic properties of vector bundles $[61,66]$. Locally, of course, $T Q$ is just $T_{g} \times \mathrm{C}^{3 g-3}$. Because $T_{g}$ is contractible, we actually have $T Q \cong T_{g} \times \mathbf{C}^{3 g-3}[61$, p. 53].

\section{Some known results}

In this brief section, we collect a number of the known results about locally schlicht L.P. functions.

THEOREM A. (Poincaré). Suppose that $z(t)$ and $w(t)$ are two linearly polymorphic functions on the same Teichmüller point $\tau \in T_{g}$. Suppose further that $m[z]=m[w]$. Then, $z(t) \equiv w(t)$.

Proof. See, for example, [5, p. 310] and [53, p. 212]. One may also refer to Theorem 15 below.

THEOREM B. (Picard). Let $z(t)$ be a linearly polymorphic function. It is impossible, then, for all the elements of $M[z]$ to have a common fixpoint.

Proof. See [5, pp. 297, 305] and [44, p. 300].

ThEOR EM C. Let $z(t)$ be a linearly polymorphic function. Then, not all of the elements of $m[z]$ can be elliptic. In particular, the marked monodromy group $m[z]$ must be infinite.

Proof. We refer to [5, pp. 62, 305].

THEOREM D. The following statements are equivalent for a linearly polymorphic func$\operatorname{tion} z(t)$ :

(i) $z(U) \neq \hat{\mathbf{C}}$

(ii) z: $U \rightarrow z(U)$ is a topological covering map;

(iii) the action of $m[z]$ on $z(U)$ is properly discontinuous.

Proof. See [18], [36], and also [5, pp. 306-310, 317-320].

Corollary. Suppose that the conditions (i)-(iii) hold. Then, $m[z]$ can be properly discontinuous in no domain strictly larger than $z(U)$.

Proof. See [36, p. 543]. 
We end this section by remarking that a useful table of invariant differential operators for various monodromy groups can be found in [33, p. 148] and [35, pp. 280-281]. Knowledge of this table can be used to motivate the proofs of Theorems $B$ and $C$.

\section{Statement of the main theorems}

The main results in this paper involve, as promised, the monodromy mapping $p: T Q \rightarrow m$ for locally schlicht L.P. functions.

We recall from section 6 that the vector bundle $T Q$ can be supplied with a very natural real-analytic structure, and that the space $m$ is contained in the complex-analytic manifold $N_{1} / L F$ :

$$
m=\frac{R}{L F(2, \mathrm{C}} \subseteq \frac{N_{1}}{L F(2, \mathrm{C})} \subseteq \frac{N}{L F(2, \mathbf{C})} \subseteq \frac{L F(2, \mathbf{C})^{2 o}}{L F(2, \mathbf{C})}
$$

THEOREM 1. The space $m$ is actually a subdomain of $N_{1} / L F$, and the mapping $p: T Q \rightarrow m$ is a local diffeomorphism.

A much weaker version of this theorem can be found in [5, pp. 335-337].

THEОR м 2. Suppose that $z(t)$ and $w(s)$ are linearly polymorphic functions (on Teichmiiller ponts $\tau_{t}$ and $\tau_{s}$ such that $m[z]=m[w]$. Then, $z(U) \cap w(U)$ is non-empty.

This result is most important as a tool to be used in studying the fibres of the mapping $p$. It enables one, for example, to get a reasonable hold on the fundamental membranes of $z$ and $w$. This particular situation will be described more precisely in section 9. Additional information about Theorem 2 can be found in [22, pp. 251-252].

In the next four theorems, we assume that the marked Fuchsian and Schottky groups referred to arise from compact Riemann surfaces of genus $g$.

THEOREM 3. Let $z(t)$ be a linearly polymorphic function such that $M[z]$ is a marked Fuchsian group element-wise. If $z(U) \neq \hat{\mathrm{C}}$, then $z(t)$ must reduce to a linear fractional transformation.

THEOREM 4. Explicit counterexamples can be constructed to show that the preceding theorem is false without the restriction $z(U) \neq \hat{\mathbf{C}}$.

THEOREM 5. Let $z(t)$ be a linearly polymorphic function such that $M[z]$ is a marked Schottky group element-wise. If $z(U) \neq \hat{\mathbf{C}}$, then $z(t)$ reduces to a Schottky uniformization. 
THEOR EM 6. The preceding theorem is false without the restriction $z(U) \neq \hat{\mathbf{C}}$.

The counterexamples alluded to in Theorems 4 and 6 show that the classical accessory parameter problems in uniformization theory have unique solutions only if the functions involved are properly restricted. Compare [13, pp. 278-279] and [47, p. 201 (problem 2)].

We conclude this section with some theorems which shed new light on the questions raised at the end of section 6 .

THEOREM 7. There exist linearly polymorphic functions $z(t)$ and $w(s)$ on Teichmüller points $\tau_{t}$ and $\tau_{s}$ such that: (i) $m[z]=m[w]$; (ii) $\tau_{t}$ and $\tau_{s}$ are conformally inequivalent as unmarked surfaces.

Consequently, the individual fibres of $\boldsymbol{p}$ are not very well behaved.

Since $p$ fails to be $1-1$, the following two results will be of interest. The first of these is actually somewhat surprising.

THEOREM 8. The mapping $p: T Q \rightarrow M$ is not a topological covering map over $M$, since there exist paths in the base space $M$ which cannot be lifted to TQ.

THEOREM 9. The mapping $p: T Q \rightarrow m$ is, however, a topological covering over the portion of $m$ which corresponds to marked quasi-Fuchsian groups of genus $g$.

Our last theorem gives important information about the size of $\mathrm{m}$.

THEOREM 10. Let $\left(L_{1}, \ldots, L_{o}\right)$ be any point of $L F(2, \mathrm{C})^{0}$ such that: (i) $L_{1}(z)=\lambda_{1} z$, $1<\left|\lambda_{1}\right|<\infty$; (ii) $L_{2}, \ldots, L_{g}$ are hyperbolic or loxodromic ; (iii) neither 0 nor $\infty$ is a fixpoint of any $L_{k}, 2 \leqslant k \leqslant g$. There then exists a linearly polymorphic function $z(t)$ on some Teichmüller point $\tau \in T_{p}$ such that $m[z]=\left[L_{1}, \ldots, L_{g} ; I, \ldots, I\right]$.

CoROLLARY. There exist monodromy groups $M[z]$ which are situated arbitrarily close to the identity group $[I, \ldots, I ; I, \ldots, I]$.

The corollary should be contrasted with [5, pp. 337-341].

Professor Schiffer has suggested that $m$ should actually be dense in $N_{1} / L F$. Theorem 10 and its corollary certainly support this view.

Finally, we remark that Theorems 1-10 were announced in [22].

\section{Proofs of the main theorems}

Before we can begin the proofs, a number of preliminary comments are necessary. We will be dealing with locally schlicht L.P. functions $z(t)$, where $t \in U / \mathcal{G}_{\tau}$ and $\tau \leftrightarrow \mathcal{G}_{\tau}$ as 
in section 6. The geometric approach to such functions is based on an important equivalence, relating L.P. functions $z(t)$ to fundamental membranes (in the sense of Klein). Both Klein and Poincaré used this equivalence in their work on monodromy groups. A glance through some of their work $[32,33,34,35,47]$ may prove enlightening here (see also [5, 27]).

There are two sides to any equivalence. We take the easy side first. Let $\mathcal{F}$ be a fundamental polygon for the marked Fuchsian $\mathcal{G}_{\tau}$. Therefore $\partial \mathcal{F}=\alpha_{1}^{+} \beta_{1}^{+} \alpha_{1}^{-} \beta_{1}^{-} \ldots \alpha_{g}^{+} \beta_{g}^{+} \alpha_{g}^{-} \beta_{g}^{-}$, where the labelling corresponds to a canonical dissection $\left\{\mathcal{A}_{k}, \mathcal{B}_{k}\right\}_{k-1}^{g}$ for the marked surface $\tau \in T_{g}$. The sides of $\mathcal{F}$ will then be identified in pairs:

$$
\left.\begin{array}{rr}
L_{k}: \alpha_{k}^{-} \rightarrow \alpha_{k}^{+}, & 1 \leqslant k \leqslant g \\
L_{\sigma+k}: \beta_{\bar{k}}^{-} \rightarrow \beta_{k}^{+}, & 1 \leqslant k \leqslant g
\end{array}\right\} .
$$

It is well-known that $[5,15,60]$ :

(i) the mappings indicated in (9.1) are orientation-reversing;

(ii) the sum of the vertex angles is $2 \pi$;

(iii) $\mathcal{G}_{\tau}=\left[L_{1}, \ldots, L_{2 g}\right]$;

(iv) the commutator relation (6.5) translates into

$$
\prod_{k=1}^{g}\left(L_{k}, L_{\sigma+k}^{-1}\right)=I
$$

It is not difficult to express the $L_{j}$ in terms of the $A_{k}$ and $B_{k}$, and vice versa.

The fundamental membrane $\mathcal{R}_{z}$ of $z(t)$ is simply the Riemannian image $z(\mathcal{F})$. The membrane $\boldsymbol{R}_{\boldsymbol{z}}$ is thus situated over the Riemann sphere $\hat{\mathbf{C}}$ and may very well be multi-sheeted. We note in particular that:

(a) $\boldsymbol{R}_{z}$ is simply-connected and has no points of ramification;

(b) any sufficiently fine triangulation of $\mathcal{I}$ yields a piecewise schlicht triangulation of $\boldsymbol{R}_{z}$;

(c) the $M[z]$ analogues of (i)-(iv) are valid;

(d) deformations of $\mathcal{F}$ induce obvious deformations of $\boldsymbol{R}_{z}$.

We now turn to the more difficult side of the equivalence. Let $\mathcal{R}$ be any simplyconnected, unramified membrane situated over $\hat{\mathbf{C}}$. Suppose that $\partial \mathcal{R}$ can be written in the form $A_{1}^{+} B_{1}^{+} A_{1}^{-} B_{1}^{-} \ldots A_{g}^{+} B_{g}^{+} A_{a}^{-} B_{g}^{-}$so that the analogues of (i)-(iv) hold for appropriate L.F. maps $T_{k}, T_{\theta+k}$. (Note that the orientation on $\hat{\mathbf{C}}$ clearly lifts to $R$.) We claim that $\mathcal{R}$ is actually a fundamental membrane $\boldsymbol{R}_{\boldsymbol{r}}$.

We examine the topological side of the question first. Choose any $\tau \in T_{g}$ and form the corresponding fundamental polygon $\mathcal{F}$. We can clearly construct an orientation-preserving local homeomorphism $f: \mathcal{F} \leftrightarrow \mathcal{R}$ which preserves the L.P. identifications along $\partial \mathcal{F}$ and $\partial \boldsymbol{R}$. 
The Fuchsian group $\mathcal{G}_{\tau}=\left[L_{1}, \ldots, L_{2 g}\right]$ is freely generated by the $L$, modulo the commutator relation (9.2). We can therefore define a homomorphism $\chi: \mathcal{G}_{\tau} \rightarrow\left[T_{1}, \ldots, T_{2 g}\right]$ by setting:

$$
\chi\left(L_{j}\right)=T_{j}, \quad 1 \leqslant j \leqslant 2 g .
$$

By means of $\chi$, we can now extend $f$ to all of $U$ :

$$
f(L t)=\chi(L)[f(t)] \text { for } L \in \mathcal{G}_{\tau}, t \in \mathcal{I}
$$

It is not difficult to check that $f$ is unambiguously defined and that (9.4) actually holds for all $t \in U$. To prove that $f$ is a local homeomorphism on $U$, we simply piece together the various Riemannian images $f[L(\mathcal{F})]$ using the analogue of (ii). This involves only a simple computation. It follows that the membranes $\chi(L) R$ can be welded together consistently, which ensures that $R=R_{f}$ for a continuous locally schlicht L.P. function $f(t)$.

The analytic side of the question is now easy. We simply identify the various sides of $\partial \mathcal{R}$ to obtain a topological surface $F$ of genus $g$. We then supply $F$ with a conformal structure by lifting the obvious one on $\hat{\mathbf{C}}$ up to $\boldsymbol{R}$. The identifications along $\partial \boldsymbol{R}$ cause only minor complications (since we know already that $\boldsymbol{R}=\boldsymbol{R}_{f}$ ). We will thus obtain a marked compact Riemann surface $\left[F,\left\{\mathcal{A}_{k}, \mathcal{B}_{k}\right\}\right]$. Let $\pi: U \rightarrow F$ be the normalized universal covering map for the marked surface $F$ and let $\eta: \hat{R} \rightarrow \hat{\mathbf{C}}$ be the obvious projection. It is apparent that we can take $\tau=F$ and $f(t)=z(t)=\eta[\pi(t)]$ in the previous two paragraphs. The function $z(t)$ will then be meromorphic on $\mathcal{F}_{F}$. By (9.4) and a classical theorem of Riemann, the extended function $z(t)$ will be meromorphic on all of $U$. It follows then that $\boldsymbol{R}=\boldsymbol{R}_{z}$, as required.

We have thus proved an equivalence between fundamental membranes $\boldsymbol{R}_{z}$ and properlyidentified membranes $R$. Recollection of (6.1) yields:

$$
\text { D.E. } \leftrightarrow z(t) \leftrightarrow R \text {. }
$$

The beauty of this three-way equivalence is that $R$ is purely geometric.

There is, however, one catch: the sides of the membrane $R$ must be identified under appropriate L.F. maps. Getting a hold of such membranes, especially the multi-sheeted ones, can be quite tricky. Furthermore, since $R$ is always subject to admissible deformations (as in (d) above), a purely analytic description of the situation seems out of the question. Compare [34, p. 39].

Armed with this preliminary information, we now turn to the proofs of Theorems 1-10.

Proof (Theorem 1). We treat the topological part of the proof first. We begin by proving that $p: T Q \rightarrow m$ is continuous at any point $\left(\tau_{0}, c_{0}\right) \in T_{g} \times \mathbf{C}^{3 g-3}$. The local identification $T Q \leftrightarrow T_{g} \times \mathbf{C}^{3 g-3}$ used here is simply:

$$
(\tau, q) \leftrightarrow\left(\tau, \sum_{k=1}^{3 g-3} c_{k} q_{k}(t ; \tau)\right) \leftrightarrow(\tau, c),
$$


in the notation of section 6 . By working locally, we can choose fundamental polygons $\mathfrak{F}_{\tau}$ for the marked Fuchsian groups $\mathcal{G}_{\tau}$, which vary continuously with $\tau$ and which are all pinned down at the same base point $t_{0} \in U$. Let $z(t ; \tau ; c)$ be the unique solution of $\{z, t\}=$ $2 q(t)$ with normalization:

$$
z(t ; \tau ; c)=t-t_{0}+O\left(t-t_{0}\right)^{s} .
$$

We can clearly factor the monodromy mapping $p$ into $p=P \circ \eta$, where

$$
(\tau, c) \stackrel{\eta}{\longrightarrow} m[z(t ; \tau ; c)] \stackrel{P}{\longrightarrow} m[z(t ; \tau ; c)] \bmod L F(2, C) .
$$

The continuity of $p$ now follows from that of $\eta$ and $P$. Note here that the smoothness of $q_{k}(t ; \tau)$ is used in proving that $\eta$ is continuous.

We next claim that $p$ is locally $1-1$. In fact, suppose that $p\left(\tau_{n}, c_{n}\right)=p\left(\sigma_{n}, d_{n}\right)$ where $\left(\tau_{n}, c_{n}\right) \neq\left(\sigma_{n}, d_{n}\right)$ and $\lim _{n \rightarrow \infty}\left(\tau_{n}, c_{n}\right)=\lim _{n \rightarrow \infty}\left(\sigma_{n}, d_{n}\right)=\left(\tau_{0}, c_{0}\right)$. Let $H_{n} m\left[z\left(t ; \tau_{n} ; c_{n}\right)\right] H_{n}^{-1}=$ $m\left[z\left(s ; \sigma_{n} ; d_{n}\right)\right]$. By passing to a possible subsequence, we may assume that either $H_{n} \rightarrow H$ in $L F(2, \mathrm{C})$ or else that $\lim _{n \rightarrow \infty} H_{n}(z)=a$ for all $z \neq b$. In the second case, we immediately see that all elements of $m\left[z\left(s ; \tau_{0} ; c_{0}\right)\right]$ must have fixpoint $a$. This contradicts theorem $B$. The first case therefore holds and, by Theorem A, we see that $H=I$.

A simple normal families argument shows that there must exist some $\delta>0$ such that the functions $z\left(t ; \tau_{n} ; c_{n}\right)$ and $z\left(s ; \sigma_{n} ; d_{n}\right)$ are all schlicht on any non-Euclidean disk of radius $\delta$. The Riemannian images $z\left(\mathcal{I} \tau_{n} ; \tau_{n} ; c_{i i}\right)$ and $z\left(\mathcal{F}_{\sigma_{n}} ; \sigma_{n} ; d_{n}\right)$ will therefore tend nicely to that of $z\left(\mathcal{I} \tau_{0} ; \tau_{0} ; c_{0}\right)$ as $n \rightarrow \infty$. The same is true for $w\left(\exists \tau_{n} ; \tau_{n} ; c_{n}\right)$, where $w\left(t ; \tau_{n} ; c_{n}\right)=H_{n}\left[z\left(t ; \tau_{n} ; c_{n}\right)\right]$. Since $m\left[w\left(t ; \tau_{n} ; c_{n}\right)\right]=m\left[z\left(s ; \sigma_{n} ; d_{n}\right)\right]$, we can distort $\mathcal{F} \tau_{i n}$ very slightly into $\overline{\mathcal{F}} \tau_{n}$ to ensure that $w\left[\overline{\mathcal{F}} \tau_{n} ; \tau_{n} ; c_{n}\right] \equiv z\left[\mathcal{F} \sigma_{n} ; \sigma_{n} ; d_{n}\right]$. By virtue of our preliminary comments (e.g. (9.5)), there will now exist an obvious ronformal mapping between $\overline{\mathcal{F}} \tau_{n}$ and $\mathcal{F} \sigma_{n}$ which preserves boundary identifications. By extending this map under the group action, we obtain a conformal homeomorphism between $\tau_{n}$ and $\sigma_{n}$ (as marked surfaces). Therefore $\tau_{n}=\sigma_{n}$ and $c_{n}=d_{n}$ (by Theorem $\mathrm{A}$ ). This is a contradiction. Hence $p$ is locally $\mathrm{l}-1$.

As noted in section 6, the ambient space $N_{1} / L F$ is a complex-analytic manifold. By applying the Brouwer theorem on invariance of domain $[4, p .156]$, the mapping $p$ is seen to be a local homeomorphism. The fact that $m$ is a subdomain of $N_{1} / L F$ is now obvious.

It remains to show that $p: T Q \rightarrow m$ is actually a local diffeomorphism. We recall that the spaces $T Q$ and $N_{1}$ were supplied with appropriate real and complex analytic structures, respectively, in section 6 . We also recall that $P: N_{1} \rightarrow N_{1} / L F$ is an analytic fibre bundle [20]. By applying (9.6)-(9.8), we immediately deduce that $p$ is real-analytic.

To complete the proof, it will be sufficient (by the implicit funotion theorem) to prove that the local inverse function $p^{-1}$ is $C^{1}$. In doing so, we may assume WLOG that the local situation reduces to that of $(9.6)-(9.8)$. 
We form the membranes $z\left(\mathcal{F}_{\tau_{0}} ; \tau_{0} ; c_{0}\right)$ and $z\left(U ; \tau_{0} ; c_{0}\right)$, and consider points $\left[X_{\alpha} ; Y_{\beta}\right] \in N_{1}$ very close to $m\left[z\left(t ; \tau_{0} ; c_{0}\right)\right]$. It is geometrically obvious that one can continuously deform the membrane $z\left(\mathcal{F}_{\tau_{0}} ; \tau_{0} ; c_{0}\right)$ within the ambient surface $z\left(U ; \tau_{0} ; c_{0}\right)$ to obtain a new membrane $\boldsymbol{R}$, whose boundary identifications correspond to $\left[X_{\alpha} ; Y_{\beta}\right]$. A simple continuity argument shows that the analogues of (i)-(iv) are valid for $\boldsymbol{R}$ (as in the preliminary comments).

We now exploit the basic geometric equivalence (9.5). By repeating the construction given near (9.3)-(9.5), we easily obtain a commutative diagram

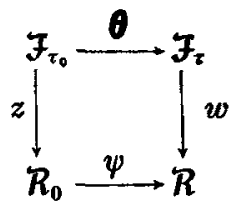

such that:

(a) $z=z(t)=z\left(t ; \tau_{0} ; c_{0}\right)$ and $\mathcal{R}_{0}=z\left(\mathcal{F}_{\tau_{0}}\right)$;

(b) $w=w(s)$ is a locally schlicht L.P. function on $\tau$ such that $\boldsymbol{R}=\boldsymbol{R}_{w}$;

(c) both $w=\psi(z)$ and $s=\theta(t)$ are orientation-preserving homeomorphisms which preserve boundary identifications;

(d) the corresponding marked Fuchsian groups $\mathcal{G}_{\tau_{0}}, \mathcal{G}_{\boldsymbol{\tau}}$ are normalized. Under the group action, $s=\theta(t)$ clearly extends to a homeomorphism $U \bmod \mathcal{G}_{\tau_{0}} \rightarrow U \bmod \mathcal{G}_{\tau}$.

By being more careful in the construction of $R$, we can certainly ensure that the mapping $\psi=\psi\left(z ; X_{\alpha} ; Y_{\beta}\right)$ is several times continuously differentiable in $\left(z ; X_{\alpha} ; Y_{\beta}\right)$. Compare [63, pp. 27-34]. The same will therefore be true for the Beltrami coefficient:

$$
\mu\left(t ; X_{\alpha} ; Y_{\beta}\right)=\frac{\theta_{t}(t)}{\theta_{t}(t)}=\frac{\psi_{z}[z(t)]}{\psi_{2}[z(t)]} \cdot \frac{\overline{z^{\prime}(t)}}{z^{\prime}(t)} .
$$

Because $s=\theta(t)$ has the fixpoint normalization of condition (d), the classical theory of the Beltrami equation $\phi_{\bar{t}}=\mu \phi_{t}$ guarantees that $s=\theta\left(t ; X_{\alpha} ; Y_{\beta}\right)$ is at least $C^{1}$ in $\left(t ; X_{\alpha} ; Y_{\beta}\right)$ $[1,3]$.

By putting this fact together with the equation $w=\psi \circ z \circ \theta^{-1}$, we see that $w\left(s ; X_{\alpha} ; Y_{\beta}\right)$ corresponds to a point of $T Q$ having a $C^{1}$ dependence on $\left[X_{\alpha} ; Y_{\beta}\right]$. Since $P: N_{1} \rightarrow N_{1} / L F$ is an analytic fibre bundle and $\left[X_{\alpha} ; Y_{\beta}\right] \in N_{1}$ is quite arbitrary, we conclude that $p^{-1}$ is $C^{\mathbf{1}}$.

It follows that $p$ is a real-analytic, local diffeomorphism.

Proof (Theorem 2). A complete proof has already been given in [22]. For the sake of completeness, we briefly recall the main ideas. One simply assumes that $z(U) \cap w(U)$ is empty and constructs the function

$$
E(t)=\frac{[w(s)-z(t)]^{2}}{w^{\prime}(s) z^{\prime}(t) \phi_{t}(t)}
$$


where $s=\phi(t)$ is any orientation-preserving diffeomorphism taking $U / \mathcal{G}_{t}$ onto $U / \mathcal{G}_{s}$. We then check that: (a) $E(t) \neq 0, \infty$; (b) $E(t)$ is differentiable; (c) $E(L t)=E(t) L^{\prime}(t)^{2}$ for $L \in \mathcal{G}_{t}$; (d) $E(t)$ is preserved under auxiliary L.F. mappings $(z, w) \rightarrow(M z, M w)$. Conditions (a)-(c) guarantee, however, that $D(t)=\sqrt{E(t)}$ induces a singularity-free vector field on either $\tau_{t}$ or an appropriate two-sheeted covering thereof (since $D(L t)= \pm D(t) L^{\prime}(t)$ ). This contradicts the classical Hopf-Poincaré index theorem [62, p. 244].

Under the hypotheses of Theorem 2, we may assume WLOG that the vertices of $z\left(\mathcal{F}_{t}\right)$ and $w\left(\mathfrak{F}_{s}\right)$ have the same respective coordinates. This is accomplished by deforming the polygons $\mathcal{F}_{t}$ and $\mathfrak{F}_{s}$. Informally expressed, the membranes $z\left(\mathcal{F}_{t}\right)$ and $w\left(\mathcal{F}_{s}\right)$ can then differ only in the way their sides are wrapped around.

Proof (Theorem 3). We may clearly apply Theorem 2 with $z=z(t)$ and (WLOG) $w=$ $w(s)=s$ to deduce that $z(U) \cap U>\phi$. Observe, however, that $m[z]=m[w]$ has region of discontinuity $\hat{\mathbf{c}}-\partial U$. By the Corollary to Theorem $\mathrm{D}$, we see that $z(U)$ must therefore coincide with $U$. An application of Theorem $\mathrm{D}$ (ii) now shows that $z(t)$ is schlicht and hence L.F.

Proof (Theorem 4). By virtue of the geometric equivalence (9.5), we need only construct an appropriate membrane $\mathcal{R}$.

To do so, let $\mathcal{F}$ be the $4 g$-sided regular N.E. polygon having center at the origin, first vertex along the positive real axis, and vertex angle $\pi / 2 g$. By a classical theorem of Poincaré $\left[60\right.$, p. 84], $\mathcal{F}$ will be the canonical polygon of some marked surface $F \in T_{g}$.

Let $\partial \mathcal{F}=\alpha_{1}^{+} \beta_{1}^{+} \alpha_{1}^{-} \beta_{1}^{-} \ldots \alpha_{g}^{+} \beta_{g}^{+} \alpha_{g}^{-} \beta_{g}^{-}$, so that (9.1) applies. We claim that the geodesics determined by the various $\alpha_{k}^{ \pm}$are well-separated. In fact, if the $\alpha_{k}^{ \pm}$geodesics are not wellseparated, they will clearly determine a $2 g$-sided regular N.E. polygon $\mathcal{D}$ which contains F. Using the Gauss-Bonnet theorem [60, 62], we see that $(4 g-4) \pi=A(\mathcal{F}) \leqslant A(\mathcal{D}) \leqslant(2 g-2) \pi$, which is a contradiction.

We now take $c_{1}$ and $c_{2}$ to be the centers of the circles determined by $\alpha_{1}^{+}$and $\alpha_{1}^{-}$, and let $W_{0}$ be the planar surface defined by $w^{2}=\left(z-c_{1}\right)\left(z-c_{2}\right)$. The Riemann surface $W_{0}$ is a two-sheeted ramified covering of $\hat{\mathbf{C}}$.

By means of the geodesics $\alpha_{k}^{ \pm}, \beta_{k}^{ \pm}$we may now define a simply-connected subregion $W$ of $W_{0}$ as depicted in figure 1 (for $g=2$ ). Because $W \subseteq W_{0}$, it certainly makes sense to write $\partial W$ and to set $\partial W=A_{1}^{+} B_{1}^{+} A_{1}^{-} B_{1}^{-} \ldots A_{g}^{+} B_{g}^{+} A_{g}^{-} B_{g}^{-}$. Corresponding to (9.1), we now have

$$
L_{k}: A_{\vec{k}} \rightarrow A_{k}^{+}, \quad L_{a+k}: B_{\vec{k}}^{-} \rightarrow B_{k}^{+} \text {. }
$$

It is clear that the membrane $W$ is properly-identified in the sense of (9.5).

One may therefore repeat the construction given near (9.3)-(9.5) to obtain a locally 


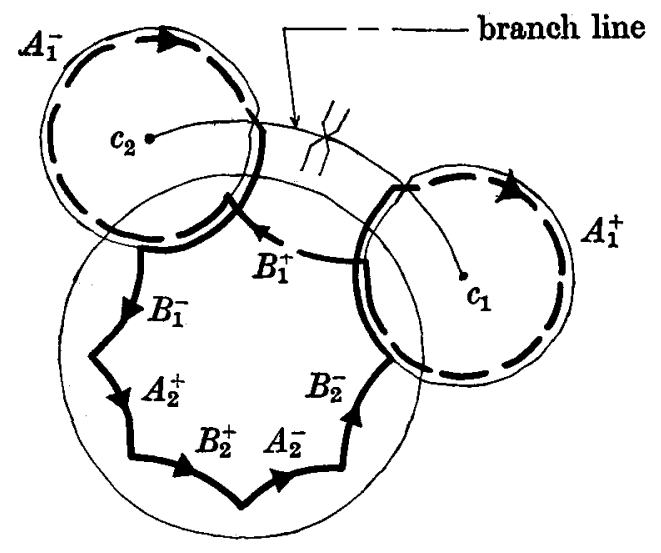

Fig. 1. - Top sheet; - - bottom sheet.

schlicht L.P. function $z(t)$, defined on some $\tau \in T_{g}$, such that $z\left(\mathcal{I}_{\tau}\right)=W$ considered as membranes.

Let $\mathcal{G}$ be the (improperly normalized) marked Fuchsian group associated with $\left[L_{1}, \ldots, L_{2 g}\right]$ and marked surface $F$. We recall (9.2). It is obvious, by construction, that $m[z]=G$. This completes the proof of Theorem 4, since the function $z(t)$ is clearly nonschlicht.

Several comments are called for here. First, the remark given after the proof of Theorem 2 is illustrated quite well by the membranes $\mathcal{I}$ and $W$ used above. Secondly, it is very easy to check directly that $z(U)=\hat{\mathbf{C}}$. And, finally, a similar example was discussed (independently) by Maskit [39, pp. 6-7]. His approach is much less geometric than ours.

Proof (Theorem 5). Assume that we are given locally schlicht L.P. functions $z$ and $w$ on marked surfaces $F$ and $H$ such that: (a) $m[z]=m[w]$; (b) $w(U) \neq \hat{\mathbf{C}}$; (c) $z: U \rightarrow z(U)$ is a Schottky uniformization. We may thus assume that the marked surface $\left[F,\left\{\mathcal{A}_{\kappa}, \mathcal{B}_{k}\right\}\right]$ has Schottky data $\left(D_{z}, S_{z}, T_{1}, \ldots, T_{g}\right)$ :

$$
\begin{array}{ll}
m[z]=\left[T_{1}, \ldots, T_{g} ; I, \ldots, I\right], & S_{z}=\left[T_{1}, \ldots, T_{g}\right], \\
z(U)=D_{z}, & F=D_{z} / S_{z} .
\end{array}
$$

The Schottky group $S_{z}$ has region of discontinuity $D_{z}$. Since $w(U) \neq \hat{\mathbf{C}}$ and $m[z]=m[w]$, the corollary to Theorem $\mathrm{D}$ shows that $w(U)=D_{2}$.

Let $\eta: U \rightarrow D_{w}$ be any Schottky uniformization for $H$. Set $H=D_{v} / S_{w}$. Using the fact that $m[w]=m[z]$, we easily see that the function $W=w \circ \eta^{-1}$ is a single-valued, locally schlicht L.P. function on $D_{w}$. More precisely,

$$
W\left[K_{\alpha} s\right]=T_{\alpha} W(s)
$$


where $1 \leqslant \alpha \leqslant g, S_{w}=\left[K_{1}, \ldots, K_{q}\right]$, and $s \in D_{w}$. By means of Theorem $\mathrm{D}$, it is not difficult to prove that $W: D_{w} \rightarrow D_{a}$ is a topological covering.

Let $D_{w}$ be a compact fundamental region for $D_{w} / S_{w}$. Then $W\left(D_{w o}\right)$ is a compact subregion of $D_{z}$. By repeated application of (9.11), it follows that $W(s) \rightarrow \partial D_{z}$ as $s \rightarrow \partial D_{w}$. Hence $W: D_{w} \rightarrow D_{z}$ is actually an $n$-sheeted covering $(n<\infty)$.

By an auxiliary L.F. mapping, we may clearly assume that $\infty \in D_{z}, \infty \in D_{w}$. Recall, however, that the singular set $E_{w}=\hat{\mathbf{c}}-D_{w}$ is an $A D$ null-set [2, chapter 4]. It follows at once that $W(s)$ remains analytic across $E_{w}$. In other words, $W(s)$ is simply a rational function of order $n$.

$W(s)$ can therefore have at most finitely many ramification points, and these must all lie in $E_{w}$. Choose any compact subset $M$ of $D_{w}$. By examining appropriate $K \in S_{w}$ and using $W(K s)=T[W(s)]$, it is not difficult to prove that $W(s)$ is actually schlicht on $K(M)$ and, hence, on $M$. Thus $n=1$ and $W(s)$ is L.F.

As a consequence, $w(t)=W[\eta(t)]$ is simply a Schottky uniformization. This completes the proof of Theorem 5. Caution: it need not be true that $F=H$ in $T_{q}$.

Before we begin the proof of Theorem 6 , it will be convenient to formulate an analogue of (9.5) for Schottky-type L.P. functions.

Suppose that $z(t)$ is a locally schlicht L.P. function on $\tau \in T_{g}$ such that $m[z]=\left[T_{1}, \ldots, T_{g}\right.$; $I, \ldots, I]$. Let $\Phi: U \rightarrow D_{\tau}$ be a Schottky uniformization for the marked surface $\tau$, and let $S_{\tau}=\left[L_{1}, \ldots, L_{g}\right]$ be the corresponding marked Schottky group. The function $Z=z \circ \Phi^{-1}$ is easily seen to be a single-valued, locally schlicht L.P. function on $D_{\tau}$ :

$$
Z\left[L_{k} s\right]=T_{k} Z(s), \quad 1 \leqslant k \leqslant g .
$$

Let $\mathcal{D}$ be any fundamental polygon for $\mathcal{S}_{\tau}$ which is bounded by $2 g$ disjoint loops $\beta_{1}^{+}, \beta_{1}^{-}, \ldots$, $\beta_{g}^{+}, \beta_{g}^{-}$such that

$$
L_{k}: \beta_{k}^{-} \rightarrow \beta_{k}^{+}, \quad 1 \leqslant k \leqslant g .
$$

The mappings indicated in (9.13) must then be orientation-reversing. The membrane $Z(D)$ has several important properties:

(i) $Z(\mathcal{D})$ is planar and has connectivity $2 g$;

(ii) $Z(D)$ is unramified;

(iii) the sides of $Z(D)$ are identified under orientation-reversing $T_{k}$.

We write $\partial Z(D)=B_{1}^{+} \cup B_{1}^{-} \cup \ldots \cup B_{g}^{+} \cup B_{g}^{-}$in an obvious notation.

To obtain the other side of the proposed equivalence, one must now mimic the arguments used near (9.3)-(9.5). We begin, of course, with a membrane $\boldsymbol{R}$ which satisfies the analogues of (i)-(iii). 
The topological part of the argument shows that $R=f(D)$ for some continuous locally schlicht L.P. function $f(s)$ on $D_{\tau}$ (where $\tau$ is arbitrary). The $\left[T_{1}, \ldots, T_{g}\right]$ images of $R$ can thus be welded together consistently to yield an unramified membrane $f\left(D_{\tau}\right)$. We let $\eta$ : $f\left(D_{\tau}\right) \rightarrow \hat{\mathbf{C}}$ be the obvious projection.

By identifying the sides of $\partial \boldsymbol{R}$ and lifting the conformal structure of $\hat{\mathbf{C}}$ up to $\boldsymbol{R}$, we clearly obtain a Riemann surface $F$ with a distinguished set of disjoint $\boldsymbol{B}_{k}$ loops. The universal covering map for $F$ factors into

$$
U \stackrel{\Phi}{\longrightarrow} D_{F} \stackrel{\pi}{\longrightarrow} F,
$$

where $\pi$ is the obvious Schottky covering and $\Phi$ is (what we have called) a Schottky uniformization map. It is easy to see that one can find a fundamental polygon $D_{F}$ for $D_{F} / S_{F}$ such that $\pi\left(D_{F}\right)=\overparen{R}$ as a membrane. As is now apparent, $Z\left(D_{F}\right)=\boldsymbol{R}$ for the locally schlicht L.P. functions $z=\eta \circ \pi \circ \Phi, Z=\eta \circ \pi$.

The loops $\mathcal{B}_{k}$ on $F$ can be deformed in many ways [23] to become part of a canonical dissection $\left\{\bar{A}_{k}, \bar{B}_{k}\right\}_{k=1}^{g}$ for $F$. Elementary considerations show that, by proper normalization of $\Phi$, one can always assume that $m[z]=\left[T_{1}, \ldots, T_{g} ; I, \ldots, I\right]$ for $\left[F,\left\{\bar{A}_{k}, \bar{B}_{k}\right\}\right]$.

We have thus proved the following Schottky-analogue of (9.5):

$$
\text { D.E. } \leftrightarrow[z(t), Z(s)] \leftrightarrow R,
$$

where the D.E. term corresponds to (6.1), and $\boldsymbol{R}$ satisfies (i)-(iii) above.

As we shall soon see, the geometric equivalence (9.15) is much easier to handle than (9.5).

Proof (Theorem 6). Because of the geometric equivalence (9.15), it will suffice to exhibit an appropriate membrane $R$. To do this, we shall exploit the same trick we used in Theorem 4. Simply take any Schottky group defined by $2 g$ circles $C_{1}^{+}, C_{1}^{-}, \ldots, C_{0}^{+}, C_{0}^{-}$which are identified pairwise under $T_{1}, \ldots, T_{g}$. To be more precise, let $C_{1}^{+}=\{|z|=3\}, C_{1}^{-}=\{|z|=1\}$, $T_{1}(z)=3 z$, and take the other $C_{k}^{ \pm}$to be small circles near $z=2$. The ambient surface $W_{0}$ in this case will correspond to $\left\{w^{2}=z\right\}$. Let $D_{k}^{ \pm}$denote the closed disk determined by $C_{k}^{ \pm}$.

The membrane $W \subseteq W_{0}$ is then defined as follows:

$$
W=\left\{W_{0} \text { restricted to } 1<|z|<3\right\}-\bigcup_{k=2}^{g}\left\{D_{k}^{ \pm} \text {in the top sheet }\right\} \text {. }
$$

We write $\partial W=B_{1}^{+} \cup B_{1}^{-} \cup \ldots \cup B_{g}^{+} \cup B_{g}^{-}$and observe that the identification $T_{1}: B_{1}^{-} \rightarrow B_{1}^{+}$can be hooked up in two ways, since $B_{1}^{+}$and $B_{1}^{-}$are two-sheeted circles. In any case, the membrane $W$ can obviously be used as $R$. See figure 2 .

Since $W$ is multi-sheeted, the corresponding L.P. function $z(t)$ cannot possibly be a Schottky uniformization. Nevertheless, $m[z]=\left[T_{1}, \ldots, T_{\theta} ; I, \ldots, I\right]$ "looks" just like a Schottky group. 


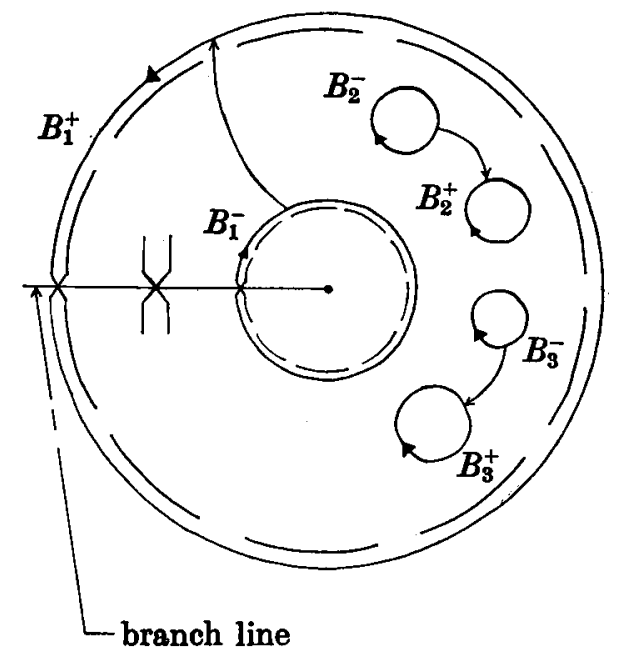

Fig. 2. $\longrightarrow$, Top sheet; - - bottom sheet.

As might be expected, it is very easy to check directly that $z(U)=\hat{\mathbf{C}}$.

Proof (Theorem 7). One is naturally tempted to use the example of either Theorem 4 or Theorem 6 in proving Theorem 7. For, in both cases, we can clearly find L.P. functions $z$ and $w$ which are quite different, yet satisfy $m[z]=m[w]$. Unfortunately, it is not clear that the associated Teichmüller points $\tau_{z}$ and $\tau_{w}$ are conformally inequivalent as unmarked surfaces.

To overcome this difficulty, one might very well try to make small perturbations in the monodromy group (based on Theorem 1). This, however, suggests an even simpler proof.

The proof presupposes some familiarity with the theory of Schottky uniformization, as in [23]. To keep matters brief, we shall be slightly informal.

Let $\Phi: T_{g} \rightarrow S_{g}$ be the mapping which associates to each $\tau \in T_{g}$ the corresponding normalized Schottky group $S_{\tau}=\left[W_{1}, \ldots, W_{0}\right]$. The normalization means that: $W_{1}(z)=\lambda_{1} z$, $0<\left|\lambda_{1}\right|<1 ; W_{2}(z)$ has attractive fixpoint $z=1$. By using the fixpoints and multipliers of the $W_{k}$, we can obviously regard $S_{g}$ as a subset of $\mathbf{C}^{3 g-3}$.

We now recall that: (a) $T_{g}$ is the universal covering of the Schottky space $S_{g}$; (b) if $\tau_{0} \in T_{g}$ has no nontrivial conformal self-maps, then the points near $\tau_{0}$ are all conformally inequivalent as unmarked surfaces.

Choose any $\tau_{1}=\left[F,\left\{\mathcal{A}_{k}, \mathcal{B}_{k}\right\}\right]$ as in (b) and apply a simple Dehn twist to get $\tau_{2}=$ $\left[F,\left\{\bar{A}_{k}, \bar{B}_{k}\right\}\right]$ with $\overline{\mathcal{A}}_{1}=\mathcal{A}_{1} \mathcal{B}_{1}, \bar{B}_{1}=\mathcal{B}_{1}, \bar{A}_{k}=\mathcal{A}_{k}, \bar{B}_{k}=\mathcal{B}_{k}$ for $2 \leqslant k \leqslant g$. The marked surfaces $\tau_{1}$ and $\tau_{2}$ have identical Schottky uniformizations: $\Phi\left(\tau_{1}\right)=\Phi\left(\tau_{2}\right)=\left[W_{1}, \ldots, W_{g}\right]$. 
We now define $B_{\lambda}(z)=\lambda z$ for $\lambda \approx 1$ and consider the locus $M$ of normalized points $\left[A_{1}, \ldots, A_{g} ; B_{\lambda}, I, \ldots, I\right] \in L F(2, \mathbf{C})^{2 g}$ with $A_{k} \approx W_{k}$. It is easy to see that $M \subseteq N_{1}$. Moreover, since $P: N_{1} \rightarrow N_{1} / L F$ is an analytic fibre bundle and the points are all normalized, the loci $M$ and $P(M)$ are biholomorphically equivalent. We may therefore identify these two loci. We observe that, since $A_{k} \approx W_{k}$ and $\lambda \approx 1$, Theorem 1 guarantees that $M \subseteq m$. There is also an obvious stratification $M=\cup M_{\lambda}$ with $\operatorname{dim}\left(M_{\lambda}\right)=3 g-3$.

Let $N\left(\tau_{j}\right) \subseteq T_{g}$ be a small neighborhood of $\tau_{j}$. By virtue of Theorem 1 , we can define a mapping $\psi_{j}: M_{\lambda} \rightarrow N\left(\tau_{j}\right)$ as follows:

$$
\left.\begin{array}{l}
\psi_{j}: M_{\lambda} \stackrel{p^{-1}}{\longrightarrow} T Q \stackrel{\text { obvious }}{\longrightarrow} T_{g} \\
\psi_{j}:\left[A_{1}, \ldots, A_{g} ; B_{\lambda}, I, \ldots, I\right] \longrightarrow \tau_{g}\left(A_{1}, \ldots, A_{g} ; \lambda\right)
\end{array}\right\}
$$

The mappings $\psi_{1}$ and $\psi_{2}$ will surely be differentiable.

We want to compare the mappings $\psi_{f}$ and $\Phi$. According to statement (a), the mapping $\Phi$ is a local homeomorphism. We maintain that $\Phi$ is actually a local diffeomorphism. This can be proved by constructing a commutative diagram (9.9) relating the fundamental polygons of marked Fuchsian and Schottky groups. To prove that $\Phi^{-1}$ is differentiable, one constructs a sufficiently smooth deformation $\psi\left[z ; X_{1}, \ldots, X_{g}\right]$ and reasons, as before, with (9.10). On the other hand, to establish that $\Phi$ is differentiable, one must begin with a sufficiently smooth deformation $\theta\left[t ; X_{1}, \ldots, X_{g} ; Y_{1}, \ldots, Y_{g}\right]$. In both cases, the groups are normalized and the classical theory of the Beltrami equation applies.

It should perhaps be remarked that by considering $S_{g}$ as a subset of $m$ and applying Theorem 1, one can prove that $\Phi^{-1}$ (and hence $\Phi$ ) is real-analytic.

We are now in a position to study the differentiable mappings $\psi_{j} \circ \Phi$. For $\lambda=1, \psi_{j} \circ \Phi$ reduces to the identity. The mappings $\psi, \circ \Phi$ and $\psi$, will therefore be local diffeomorphisms as $\lambda \rightarrow 1$.

Suppose, however, that $\tau_{1}\left(A_{1}, \ldots, A_{g} ; \lambda\right)$ and $\tau_{2}\left(A_{1}, \ldots, A_{g} ; \lambda\right)$ are always conformally equivalent as unmarked surfaces. A standard normal families argument [using (b)] shows that WLOG these Teichmüller points differ only by the simple Dehn twist cited above.

Consider now the obvious I.P. function $z_{1}$ associated with $\tau_{1}\left(A_{1}, \ldots, A_{g} ; \lambda\right)$. It is easy to check (using continuity) that, when looked at on $\tau_{2}\left(A_{1}, \ldots, A_{g} ; \lambda\right)$, the monodromy group of $z_{1}$ is simply $\left[A_{1} B_{\lambda}, A_{2}, \ldots, A_{g} ; B_{\lambda}, I, \ldots, I\right]$. This group certainly lies in $M_{\lambda}$. Therefore

$$
\psi_{2}\left[A_{1} B_{\lambda}, A_{2}, \ldots, A_{g} ; B_{\lambda}, I, \ldots, I\right]=\psi_{2}\left[A_{1}, \ldots, A_{g} ; B_{\lambda}, I, \ldots, I\right]=\tau_{2}\left(A_{1}, \ldots, A_{g} ; \lambda\right) .
$$

Since $\psi_{2}$ is a local diffeomorphism, we obtain the contradiction $A_{1} B_{\lambda}=A_{1}$ by taking $\lambda \neq 1$.

Theorem 7 , with $m[z]=m[w]=\left[A_{1}, \ldots, A_{g} ; B_{\lambda}, I, \ldots, I\right]$, follows at once.

Proof (Theorem 8). A proof valid for $g \geqslant 3$ was sketched in [22]. Unfortunately, the 


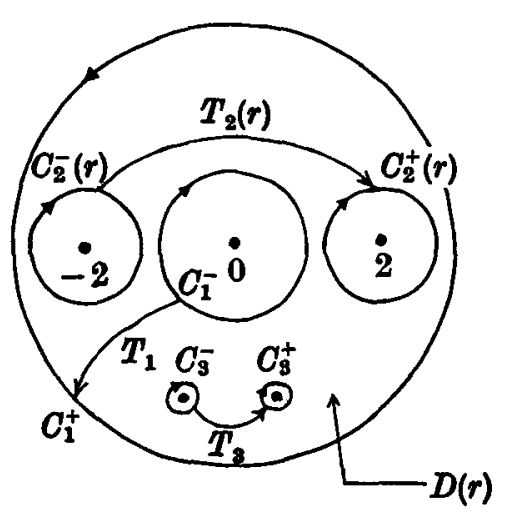

Fig. 3

role of geometric equivalence (9.15) was not made very clear there. We shall therefore reexamine the situation here in somewhat greater detail.

Suppose at first that $g \geqslant 3$. For simplicity, one may assume that $g=3$. We define a circular domain $D(r)$ for $0<r<1$ as shown in figure 3. We take $C_{1}^{-}=\{|z|=1\}, C_{1}^{+}=$ $\{|z|=3\}, \quad C_{2}^{-}(r)=\{|z+2|=r\}, C_{2}^{+}(r)=\{|z-2|=r\}, C_{3}^{-}=\{|z-a|=\delta\}, C_{3}^{+}=\{|z-b|=\delta\}$, and set

$$
T_{1}(z)=3 z, T_{2}(r)(z)=2-\frac{r^{2}}{z+2}, T_{3}(z)=b+\frac{\delta^{2}}{z-a}
$$

The region $D(r)$ obviously defines a Schottky group $\left[T_{1}, T_{8}(r), T_{\mathrm{s}}\right]$. Using (9.15) and Theorem 1, we will clearly obtain a family of L.P. functions $z_{r}$ such that: (a) $m\left[z_{r}\right]=\left[T_{1}, T_{2}(r)\right.$, $\left.T_{3} ; I, I, I\right]$; (b) $z_{r}$ is a Schottky uniformization; (c) the corresponding points of $T Q$ vary continuously with $r$.

Let $\tau(r)$ be the point of $T_{g}$ which corresponds to $z_{r}$. We maintain that $\tau(r) \rightarrow \partial T_{g}$ as $r \rightarrow 1$. If not, we could then find $r_{n} \uparrow 1$ such that $\tau\left(r_{n}\right) \rightarrow \tau_{0}$ in $T_{g}$. Recall, however, the universal covering map $\Phi: T_{o} \rightarrow S_{g}$ used in Theorem 7 (proof). Surely $\lim _{n \rightarrow \infty} \Phi\left[\tau\left(r_{n}\right)\right]=\Phi\left[\tau_{0}\right]$. But, modulo a well-behaved normalization, $\Phi\left[\tau\left(r_{n}\right)\right]=\left[T_{1}, T_{2}\left(r_{n}\right), T_{3}\right]$. It follows therefore that $\left[T_{1}, T_{2}(1), T_{3}\right]$ must be a well-defined Schottky group. This is a contradiction, since the commutator $\left(T_{1}, T_{2}(1)\right)$ is parabolic.

We now construct an analogous circular subregion $\mathcal{D}(r)$ on the planar surface $W_{0}$ : $w^{5}=(z-a)^{2}(z-b)^{3}$. The boundary components $B_{1}^{-}, B_{1}^{+}, B_{2}^{-}(r), B_{2}^{+}(r)$ are situated over $C_{1}^{-}, C_{1}^{+}$, $C_{2}^{-}(r), C_{2}^{+}(r)$, respectively, and are placed in separate sheets. The components $B_{3}^{-}$and $B_{3}^{+}$ are situated over $C_{3}^{-}$and $C_{3}^{+}$, and revolve around the branch points $z=a, z=b$ in an obvious way. See figure 4.

As in [22], we identify the various components of $\partial \mathcal{D}(r)$ under $T_{1}, T_{2}(r), T_{3}$. Since the ambient surface $W_{0}$ is planar, the subregion $\mathcal{D}(r)$ can clearly be used as a membrane $R$ 


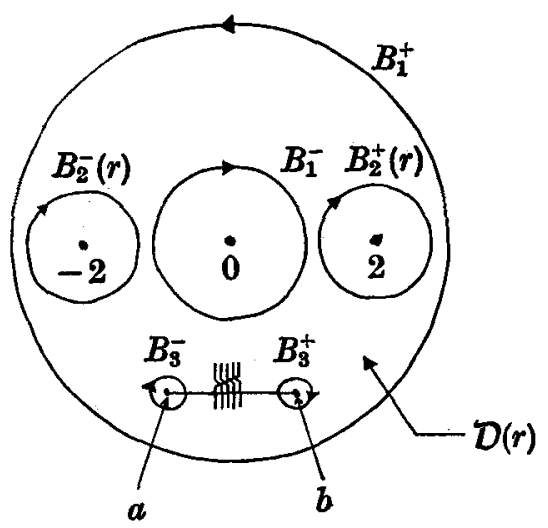

Fig. 4

in (9.15). By means of Theorem 1 and (9.15), we will thus generate a family of L.P. functions $w_{r}$ such that: (a) $M\left[w_{r}\right]=\left[T_{1}, T_{2}(r), T_{3} ; I, I, I\right]$; (b) $w_{r}$ is not a Schottky uniformization; (c) the corresponding points of $T Q$ vary continuously with $r$.

Observe, however, that the membrane $\mathcal{D}(r)$ and the construction of $w_{r}$ both remain perfectly well-defined for $r$ slightly larger than 1 . The path in $m$ corresponding to [ $T_{1}$, $\left.T_{2}(r), T_{3} ; I, I, I\right]$ for $1-\delta \leqslant r \leqslant 1+\delta$ can therefore be used to prove Theorem 8 when $g=3$, as is seen by comparing the properties of $z_{r}$ and $w_{r}$. The case $g \geqslant 4$ is handled by adding more circles.

To handle the case $g=2$, one uses the circles $C_{1}^{-}, C_{1}^{+}, C_{2}^{-}(r), C_{2}^{+}(r)$ to define $D(r)$. The region $\mathcal{D}(r)$ is then taken on the Riemann surface $w^{2}=z$ very much like before, except that $B_{1}^{-}$and $B_{1}^{+}$must be bent as shown in figure 5 .

Proof (Theorem 9). We begin by considering those $\tau \in T_{g}$ and $q(t ; \tau) \in Q[\tau]$ such that $\{z, t\}=2 q(t ; \tau)$ defines a quasi-Fuchsian uniformization. In this context, see [12, pp. 575$581,588],[13$, pp. 277-279, 294]. The corresponding subset $B$ of $T Q$ will have fibres $B(\tau)$ which are identifiable with $T_{g}$. Since the fibres of $B(\tau)$ are contractible, so is $B[61, \mathrm{pp} .53$, 90]. The quasi-Fuchsian part of $m$ is naturally defined to be the domain $m_{q}=p[B]$.

By a simple extension of the proof to Theorem 3, one may check that $p: B \rightarrow m_{q}$ is a homeomorphism. There is only one small complication. If $m[z]=m[w]$ for quasi-Fuchsian uniformizations $z$ and $w$, then we must now show that the fundamental membranes $\boldsymbol{R}_{z}$ and $\boldsymbol{R}_{w}$ can actually be taken identical. In view of the remarks following Theorem 2 (proof), this is not difficult. See also [23, sections 4 and 5].

We want to show that $p$ is a topological covering over $m_{q}$. Since $p: T Q \rightarrow m$ is a loca homeomorphism, it will suffice to prove the path-lifting property. We therefore let $\gamma=$ 3-752905 Acta mathematica 135. Imprimé le 19 Décembre 1975 


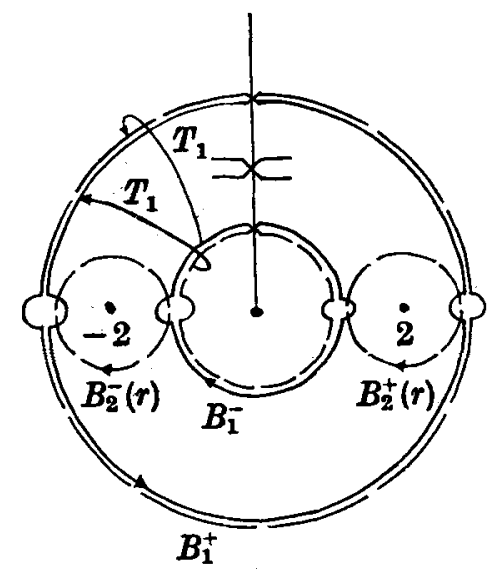

Fig. 5. - Top sheet; - - bottom sheet.

$\{M(\xi): 0 \leqslant \xi \leqslant 1, \xi \nearrow\}$ be any continuous path in $m_{q}$. WLOG $p^{-1}(\gamma) \subseteq B$ is well-inside a coordinate patch for $T Q: p^{-1}[M(\xi)]=[\tau(\xi), c(\xi)] \in T_{0} \times C^{80-8}$.

$T_{o}$ can be thought of as the set of deformations $[F, f]$ of a fixed reference surface $\left[\boldsymbol{F}_{0},\left\{\mathcal{A}_{k}, \mathcal{B}_{k}\right\}\right]$, where $f: F_{0} \rightarrow F$. See [13]. We shall therefore write $\tau(\xi)=\left[F_{\xi}, f_{\xi}\right]$. The map $f_{\xi}$ lifts to $\varphi_{\xi}: U \rightarrow U$. Because $f_{\xi}$ is determined only up to isotopy and $M(\xi)$ is continuous, WLOG $\varphi_{\xi}(t)$ is continuous on $U \times[0,1]$. The corresponding normalized Fuchsian group will then be $\mathcal{G}_{\xi}=\left[A_{1, \xi}, \ldots, B_{0, \xi}\right]$ with fundamental polygon $\mathcal{F}_{\xi}=\varphi_{\xi}(\mathcal{F})$.

We now consider the Schwarzian D.E.

$$
\{z, t\}=2 \sum_{k=1}^{30-3} c_{k}(\xi) q_{k}[t ; \tau(\xi)]
$$

This D.E. obviously defines a normalized quasi-Fuchsian function $z=z_{\xi}(t)$ such that $z=t+O\left(t^{3}\right)$. As usual then [12, pp. 588-589]: (i) $z_{\xi}(t)$ extends to a $Q C$ self-mapping of $\hat{\mathbf{c}}$; (ii) $z_{\xi} \circ A_{k, \xi}=\tilde{A}_{k, \xi} \circ z_{\xi}, z_{\xi} \circ B_{k, \xi}=\tilde{B}_{k, \xi} \circ z_{\xi} ;$ (iii) $m\left[z_{\xi}\right]=\left[\tilde{A}_{1, \xi}, \ldots, \tilde{B}_{0, \xi}\right]$ is continuous in $\xi$ and projects onto $M(\xi)$; (iv) $z_{\xi}(t)$ is continuous on $\hat{\mathbf{C}} \times[0,1]$. See also [13, section 2.5].

Define $\psi_{\xi}=\varphi_{\xi} \circ \varphi_{0}^{-1}$. This function satisfies $\psi_{\xi} \mathcal{G}_{0} \psi_{\xi}^{-1}=\mathcal{G}_{\xi}$ and is continuous on $U \times$ $[0,1]$. By reflection, $\psi_{\xi}$ extends to a $Q C$ self-map of $\hat{\mathbf{c}}$. By adjusting the $f_{\xi}$ and using the Beltrami equation, we easily see that $\psi_{\xi}(t)$ is continuous on $\hat{\mathbf{C}} \times[0,1]$.

Suppose now that $m[w]=\left[\tilde{A}_{1,0}, \ldots, \tilde{B}_{g, 0}\right]$. We must try to lift the path $\gamma$ through $w$. Certainly, by the above-mentioned extension of Theorem 3, one may assume WLOG that $w(U)=\hat{\mathbf{C}}$. We let $w=w(s)$ correspond to $[G, h] \in T_{g}$ with normalized Fuchsian data $[\mathcal{G}, \mathcal{F}$, $\left.A_{k}, B_{k}\right]$. By Theorem 2, we may assume that the vertices of $w(\mathcal{F})$ and $z_{0}\left(\mathcal{F}_{0}\right)$ have identical coordinates. 
The mapping $\Phi_{\xi}=z_{\xi} \psi_{\xi} z_{0}^{-1}$ is a $Q C$ self-mapping of $\hat{\mathrm{C}}$ taking $z_{0}(U)$ onto $z_{\xi}(U)$. The $\Phi_{\xi}$ are certainly continuous in $\xi$ and will satisfy

$$
\Phi_{\xi} \circ \tilde{A}_{k, 0}=\tilde{A}_{k, \xi} \circ \Phi_{\xi}, \quad \Phi_{\xi} \circ \tilde{B}_{k, 0}=\tilde{B}_{k, \xi} \circ \Phi_{\xi} .
$$

The trick is to study the Riemannian images $\Phi_{\xi} w(\mathcal{F})$ for $0 \leqslant \xi \leqslant 1$. The case $\xi=0$ is obvious, since $\Phi_{0}=I$. Observe, however, that $\Phi_{\xi} w$ is a non-analytic locally schlicht L.P. function:

$$
\Phi_{\xi} w \circ A_{k}=\tilde{A}_{k, \xi} \circ \Phi_{\xi} w, \quad \Phi_{\xi} w \circ B_{k}=\tilde{B}_{k, \xi} \circ \Phi_{\xi} w .
$$

Therefore $\Phi_{\xi} w(\mathcal{I})$ defines a fundamental membrane for some locally schlicht L.P. function having monodromy group $\left[\tilde{A}_{1, \xi}, \ldots, \tilde{B}_{g . \xi}\right]$. We recall (9.5) here. By using Theorem 1 and the continuity in $\xi$, we immediately obtain the required lifting of $\gamma$. This completes the proof.

Proof (Theorem 10). It will be convenient to break up this proof into a series of steps. The proof is essentially a "packing" problem with L.F. identifications.

(I) Let $L$ be any loxodromic or hyperbolic L.F. map such that $L(0) \neq 0, L(\infty) \neq \infty$. One can then find two Jordan regions $R_{1}$ and $R_{2}$ such that: (i) $R_{1} \subseteq \hat{\mathbf{C}}-\{0, \infty\}$; (ii) $R_{2} \subseteq \hat{\mathbf{C}}-$ $\{0, \infty\}$; (iii) $L$ [int $\left.R_{1}\right]=\operatorname{ext} R_{2}$. We do not assert that $R_{1}$ and $R_{2}$ are disjoint.

Proof. Let $a, b$ be the attractive, repulsive fixpoints of $L: a, b \neq 0, \infty$. Consider the cyclic group $[L]$ acting on $D=\hat{\mathbf{C}}-\{a, b\}$. The action is properly discontinuous [15, pp. 52-54, 146-147]. As usual, $F=D /[L]$ is a compact Riemann surface of genus 1 and the projection $\pi: D \rightarrow F$ is simply the Schottky covering map.

We can therefore choose two disjoint Jordan curves $C_{1}$ and $C_{2}$ whose exterior is a fundamental region for $D /[L]$. WLOG $b \in \operatorname{int} C_{1}, a \in \operatorname{int} C_{2}$. The curves $C_{1}$ and $C_{2}$ can of course be subjected to small deformations.

There are three cases to consider: (i) $0 \in \operatorname{int} C_{1}$; (ii) $0 \in \operatorname{int} C_{2}$; (iii) $0 \in\left(\exp C_{1}\right) \cap\left(\operatorname{ext} C_{2}\right)$. The analysis for (iii) is obvious.

Consider case (i). We can continuously deform $C_{1}$ into a Jordan curve $C_{1}^{\prime}$ with $0 \in \operatorname{ext} C_{1}^{\prime}$ by means of an isotopy on $D$ which avoids $L^{-1}(0)$ and $L^{-1}(\infty)$. Applying $L$, we obtain an isotopy of $C_{2}$ into $C_{2}^{\prime}$ on $D$ which avoids 0 and $\infty$. Therefore, $0 \in \operatorname{ext} C_{2}^{\prime}$. Obviously, then, $R_{1}=\operatorname{int} C_{1}^{\prime}, R_{2}=\operatorname{int} C_{2}^{\prime}$ will work.

Case (ii) is handled similarly via $L^{-1}$.

(II) Let $\omega>0$. We claim that there exist arbitrarily large integers $N$ such that the fractional part of $N \omega / 2 \pi$ is either 0 or else very close to 1 .

Proof. When $\omega / 2 \pi$ is rational, the result is trivial. For irrational $\omega / 2 \pi$, one simply uses the classical uniform distribution theorem of $\mathbf{H}$. Weyl. 
(III) We now recall the notation used in stating Theorem 10 . We shall write $\lambda_{1}=R e^{i \omega}$, where $R=\left|\lambda_{1}\right|>1$ and $2 M \pi \leqslant \omega<(2 M+2) \pi$. $M$ is understood to be a large integer, dependent only on $g$, which will be specified later. We shall also choose $\eta$ near to 1 so that $0<R^{-1}<\eta<1$.

We must now construct the following two sequences $\bmod N$, where $N$ is any large integer.

\begin{tabular}{|c|c|c|c|}
\hline$n$ & $c_{n}$ & $d_{n}$ & \\
\hline 0 & $\frac{1}{\eta}(\eta R)^{N}$ & 1 & \\
\hline 1 & $\frac{1}{\eta}(\eta R)$ & $\eta R$ & \\
\hline 2 & $\frac{1}{\eta}(\eta R)^{2}$ & $(\eta R)^{2}$ & \\
\hline$\vdots$ & $\vdots$ & $\vdots$ & \\
\hline$N-1$ & $\frac{1}{\eta}(\eta R)^{N-1}$ & $(\eta R)^{N-1}$ & $\left(c_{n+1}=R d_{n}\right)$ \\
\hline$N$ & $\frac{1}{\eta}(\eta R)^{N}$ & 1 & $\left\{\begin{array}{l}\eta<1 \\
\eta R>1\end{array}\right\}$ \\
\hline
\end{tabular}

In an obvious way, $\left\{c_{n}\right\}_{n=0}^{N}$ determines a polygonal function $y=c(x)$ on $0 \leqslant x \leqslant N$.

Consider now any $\alpha: N \leqslant \alpha<N+\frac{1}{2}$. We define $c(x)$ on $[0, \alpha]$ via

$$
c(x)=\left\{\begin{array}{l}
c(x), \quad 0 \leqslant x \leqslant N, \\
\frac{1}{\eta}(\eta R)^{N}, \quad N \leqslant x \leqslant \alpha,
\end{array}\right.
$$

and then extend $c(x)$ to all $x$ by periodicity. Clearly $y=c(x)$ is a polygonal function; it is also easily seen that $c(x) \geqslant R$.

We next define

$$
d(x)=\frac{c(x+1)}{R}
$$

Obviously $d(x)$ is polygonal, has period $\alpha$, and satisfies $d(x) \geqslant 1$.

(IV) We claim that $c(x)>d(x)$ for all $x$.

Proof. WLOG $0 \leqslant x \leqslant \alpha$. Moreover, since $c(x)$ and $d(x)$ are piecewise-linear, we need only check the vertices: $x=0,1,2, \ldots, N-1, \alpha-1, N, \alpha$. The computations called for are all quite easy. 
(V) From now on, we restrict our attention to very large $N$ which satisfy

$$
\frac{N \omega}{2 \pi}=K-\delta, K \in \mathbf{Z}, 0 \leqslant \delta<\frac{1}{10} .
$$

For these $N$, we put $\alpha=2 \pi K / \omega$. (Recall $\omega$ in step III.) It is simple to check that

$$
0 \leqslant \alpha-N<\frac{1}{10 M}<\frac{1}{10} \text {. }
$$

(VI) Let us temporarily fix any such $N$ and consider the compact Riemann surface $\boldsymbol{F}_{\mathbf{0}}$ defined by $w^{K}=z$. The branch line is taken to be the positive real axis and the sheets are labelled cyclically: $1,2, \ldots, K . F_{0}$ is seen to be planar.

We then lift the following two curves to $F_{0}$ :

$$
z=c(x) e^{i \omega x}, \quad z=d(x) e^{i \omega x}, \quad 0 \leqslant x \leqslant \alpha .
$$

Both lifts are started in sheet 1 . Since $1 \leqslant d(x)<c(x)$, we clearly trace out an annulus $F_{1}$ on $F_{0}(\omega \alpha=2 \pi K$ by construction).

This annulus $F_{1}$ has some very important properties. In particular, consider the lifts for $0 \leqslant x \leqslant \frac{1}{2}$. We easily check that:

$$
\frac{1}{2 \eta}(\eta R)^{N} \leqslant c(x) \leqslant \frac{1}{\eta}(\eta R)^{N}, \quad 1 \leqslant d(x) \leqslant \eta R, \quad 0 \leqslant x \leqslant 1 / 2 .
$$

Thus $d(x)$ is relatively small, while $c(x)$ is enormous ( $N$ large).

The part of the annulus $F_{1}$ which corresponds to $0 \leqslant x \leqslant 1 / 2$ subtends an angle $\frac{1}{2} \omega$ :

$$
M \pi \leqslant \frac{1}{2} \omega<(M+1) \pi \text {. }
$$

The number $M$ can now be chosen: we shall set

$$
M=100 g \text {. }
$$

The portion of $F_{1}$ due to $0 \leqslant x \leqslant \frac{1}{2}$ thus lies in at least the sheets $1,2, \ldots, 50 g$. And, of course, $F_{1}$ is very wide here.

(VII) We must now apply step I to the L.F. maps $L_{2}, \ldots, L_{g}$ separately. We thus ob. tain Jordan regions $R_{2}^{\prime}, R_{2}^{\prime \prime}, \ldots, R_{g}^{\prime}, R_{g}^{\prime \prime}$. Let $h>0$ satisfy

$$
\{|z| \leqslant h\} \subseteq \bigcap_{k=2}^{0}\left[\left(\operatorname{ext} R_{k}^{\prime}\right) \cap\left(\operatorname{ext} R_{k}^{\prime \prime}\right)\right] .
$$

Of course, $h$ is determined solely by the initial data and is independent of $N$.

It is now necessary to shrink $F_{1}$ by a certain factor $C<1$ chosen so that $C \eta R<h$; we obtain the annulus $F_{2}=C F_{1}$.

Since $N$ is very large and $C d(x) \leqslant C \eta R<h$ on $0 \leqslant x \leqslant \frac{1}{2}$, we can now make the following deletions from $F_{2}$ : 
$R_{2}^{\prime}$ from sheets 1 and 2;

$R_{2}^{\prime \prime}$ from sheets 3 and 4;

$R_{g}^{\prime}$ from sheets $2 g-3$ and $2 g-2$;

$R_{g}^{\prime \prime}$ from sheets $2 g-1$ and $2 g$.

In this way we obtain a planar surface $F_{8} \subseteq F_{0}$ of connectivity $2 g$ :

$$
\partial F_{3}=\beta_{1}^{-} \cup \beta_{1}^{+} \cup \ldots \cup \beta_{g}^{-} \cup \beta_{g}^{+} \text {. }
$$

The shores $\beta_{k}^{-}$and $\beta_{k}^{+}$for $k \geqslant 2$ are clearly identified under $L_{k}$.

The shores of $\partial F_{1}$ are identified under $L_{1}(z)=\lambda_{1} z$ as follows:

$$
d(x) e^{i \omega x} \rightarrow c(x+1) e^{i \omega x(x+1)} \text {. }
$$

This correspondence holds for all $x$. There is obviously a similar correspondence for $\partial F_{2}=$ $\beta_{1}^{-} \cup \beta_{1}^{+}$.

(VIII) Taken with these identifications, $F_{3}$ clearly defines an admissible membrane $\boldsymbol{R}$ in the geometric equivalence (9.15). There will thus exist an L.P. function $z$ on some $\tau \in T_{g}$ so that

$$
m[z]=\left[L_{1}, \ldots, L_{g} ; I, \ldots, I\right]
$$

This completes the proof.

Theorem 10 is clearly a first step in determining the exact size of $\mathrm{m}$. Its proof was reduced, in essence, to a packing problem by virtue of (9.15): we merely had to construct an appropriate membrane $\boldsymbol{R}$. The construction given above was relatively transparent, because one needed to pack only the $B_{k}^{ \pm}$components and, then, only in a more-or-less independent fashion (recall steps I, VII).

In the general case, however, $m[z]=\left[X_{1}, \ldots, X_{g} ; Y_{1}, \ldots, Y_{g}\right]$ and one is forced to use (9.5). The problem is thereby reduced to constructing an appropriate simply-connected membrane $R$. The construction of $\boldsymbol{R}$ in this case is much less obvious than before. There are two major difficulties:

(a) the components $A_{k}^{ \pm}$and $B_{k}^{ \pm}$will, in general, be interlocked nontrivially. For this reason, they must be packed into $R$ simultaneously.

(b) the $A_{k}^{ \pm}$and $B_{k}^{ \pm}$can wiggle very severely. This makes it difficult to choose the correct ambient surface $W_{0}$ in advance.

It is precisely these geometric difficulties which stand in the way of proving, for example, that $m$ is dense in $N_{1} / L F$. 


\section{Some further results}

In this section, we take up several results which are closely related to those given in sections 7-9.

THEOREM 11. On any marked surface $\tau \in T_{g}$, there exist locally schlicht L.P. functions $z(t)$ whose monodromy group $M[z]$ contains infinitesimal elements.

Proof. Observe that, in the notation of (4.6) and (6.8), $\operatorname{Tr}^{2}\left(\tilde{B}_{1}\right)$ is an entire function of the accessory parameters $\lambda_{1}, \ldots, \lambda_{3 g-3}$. This function is surely non-constant, as is seen by using the Fuchsian and Schottky uniformizations. We can therefore apply the classical Picard theorem (for entire functions) to ensure that $\tilde{B}_{1}$ is frequently an elliptic element of infinite order. This suffices to prove the theorem. See also [5, p. 319 (top)], [36, p. 545].

Explicit examples can easily be constructed along the lines of figure 2 in Theorem 6. The circles $C_{2}^{-}$and $C_{2}^{+}$are taken to be isometric circles corresponding to an elliptic element $T_{2}$ and are placed in opposite sheets [15, p. 27].

Suppose next that $z(t)$ and $w(t)$ are locally schlicht L.P. functions (on the same $\tau \in T_{g}$ ) such that $m[z]=\left[A_{1}, \ldots, A_{g} ; B_{1}, \ldots, B_{g}\right]$ and $m[w]=\left[C_{1}, \ldots, C_{g} ; B_{1}, \ldots, B_{g}\right]$. We want to investigate the relation between $z$ and $w$. The global situation is definitely not immediate; e.g. consider $\left(B_{1}, \ldots, B_{g}\right)=(I, \ldots, I), z(t)=$ Schottky uniformization, and $w(t)=$ the function developed for Theorem 6.

Observe, however, that the monodromy space $m(\tau)$ (which corresponds to L.P. functions on $\tau$ ) is a $3 g-3$ dimensional submanifold of $m$. Suppose, for a moment, $\operatorname{that}\left(B_{1}, \ldots, B_{g}\right)$ were normalized to exclude the trivial case $w=T z, T \in L F(2, \mathrm{C})$. Such $\left(B_{1}, \ldots, B_{g}\right)$ depend upon $3 g-3$ parameters. By counting the constants, one would therefore expect that jocally we could assert that $z(t)=w(t)$. The corresponding assertion for unnormalized $B_{k}$ would be that $w=T z$.

The following result may therefore be of some interest.

THEOREM 12. Let the situation of the previous discussion apply. If $z(t)$ is the classical Schottky uniformization map with $m[z]=\left[A_{1}, \ldots, A_{j} ; I, \ldots, I\right]$, and if $w(t)$ is sufficiently close to $z(t)$ in $T Q$, then $w(t)=T[z(t)]$ for some $T \in L F(2, \mathrm{C})$.

Proof. Let $\{z, t\}=q(t)$ and $\{w, t\}=r(t)$, so that $q(t) \approx r(t)$. Let $z(t ; h)$ be the solution of $\{z, t\}=(1-h) q(t)+h r(t), 0 \leqslant h \leqslant 1$, normalized via $z=t+O\left(t^{3}\right)$. The fundamental membrane $z(\mathcal{F} ; h)$ varies continuously. Since $q(t) \approx r(t)$, we immediately deduce that the Riemannian paths $z\left(\beta_{k}^{ \pm} ; 1\right)$ are actually Jordan curves situated very close to $z\left(\beta_{k}^{ \pm} ; 0\right)$. Here $\partial \mathcal{I}=$ $\alpha_{1}^{+} \beta_{1}^{+} \alpha_{1}^{-} \beta_{1}^{-} \ldots \alpha_{g}^{+} \beta_{g}^{+} \alpha_{g}^{-} \beta_{g}^{-}$, as in (9.1). Elementary use of the argument principle will now 
show that the Riemannian image $z(F ; 1)$ is actually schlicht and that it is, in fact, a fundamental region for a Schottky group. By using the group action and elementary uniqueness properties for Schottky uniformization, we conclude that $z(t ; 0)=z(t ; 1)$, whence $w(t)=T[z(t)]$

The third result to be considered concerns the behavior of locally schlicht L.P. functions with respect to a regular (or normal) topological covering $\xi: F \rightarrow F$ with sheet number $n[2,29]$. The universal covering map $\pi: U \rightarrow F$ now factors into $\pi=\xi \circ \varphi$, where $\varphi: U \rightarrow \tilde{F}$. The corresponding automorphic groups $\mathcal{G}_{F}$ and $\mathcal{G}_{\bar{F}}$ will satisfy $\mathcal{G}_{\tilde{F}} \leqslant \mathcal{G}_{F}$ (normal subgroup) with $\left[\mathcal{G}_{F}: \mathcal{G}_{\tilde{F}}\right]=n$. The Euler characteristics will satisfy $\chi(\tilde{F})=n \chi(F)$ so that

$$
\tilde{g}=1+n(g-1), \quad g \geqslant 2 .
$$

In an obvious way, then, any L.P. function $z(t)$ on $\left(U, \pi, \mathcal{G}_{F}\right)$ becomes an L.P. function on $\left(U, \varphi, G_{\tilde{F}}\right)$. Because of complications in the topology, that is, in the actual canonical dissections, there seems to be no simple formula relating $m_{F}[z]$ and $m_{\tilde{F}}[z]$.

Let us now fix the L.P. function $z=z(t)$ and introduce the usual monodromy homomorphism $L \rightarrow L$ taking $\mathcal{G}_{F} \rightarrow L F(2, \mathrm{C})$. If $\gamma$ is any closed path on $F$, then the $(U, \pi)$ lifts $\tilde{\gamma}$ of $\gamma$ are all equivalent $\bmod \mathcal{G}_{F}$. To be precise, let some choice of $\tilde{\gamma}$ start at $t_{1}$ and terminate at $N t_{1}$. We can then legitimately define

$$
\boldsymbol{M}(\gamma)=\tilde{N} \bmod m_{F}[z] .
$$

The mod $m_{r}[z]$ refers to inner automorphisms. The quantity $M(\gamma)$ is clearly invariant under continuous deformation (free homotopy) of $\gamma$.

We claim that by choosing $z, F, F$ appropriately, one can construct some very interesting monodromy groups.

For example, assume that $g=3$ and start off with the situation depicted in figure 6 .

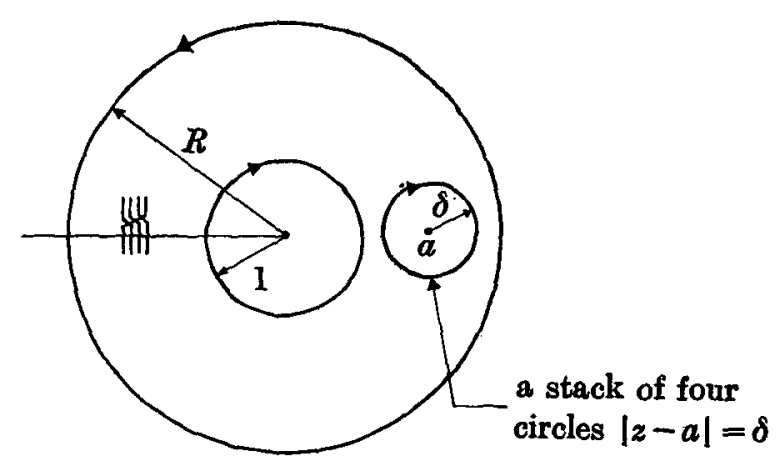

Fig. 6 
As in section 9 , one can then construct $F$ and $z$ so that

$$
\begin{gathered}
m[z]=\left[T_{1}, T_{2}, T_{3} ; I, I, I\right], \\
T_{1}(w)=R w, R>1 ; \quad T_{2}(w)=T_{3}(w)=a+\frac{\delta^{2}}{w-a}, \\
T_{2}^{2}=T_{3}^{2}=I .
\end{gathered}
$$

In what follows, we must distinguish between a canonical dissection $\left\{\mathcal{A}_{k}, \mathcal{B}_{k}\right\}_{k=1}^{o}$ and a canonical homology basis $\left\{\alpha_{k}, \beta_{k}\right\}_{k=1}^{g}$. In a dissection, the various cuts all pass through the same base point $O$. In a homology basis, however, the pairs of conjugate cuts are wellseparated from each other. Of course, by adding simple paths $c$, from $O$ to $\alpha_{j}^{+} \cap \beta_{j}^{-}$we can deform $\left\{\alpha_{k}, \beta_{k}\right\}_{k=1}^{g}$ into a canonical dissection $\left\{\mathcal{A}_{k}, \boldsymbol{B}_{k}\right\}_{k=1}^{g}$.

Consider the character $\chi$ on $\pi_{1}(F, O)$ defined by

$$
\begin{aligned}
& \chi\left(\mathcal{A}_{1}\right)=+1, \quad \chi\left(\mathcal{A}_{2}\right)=-1, \quad \chi\left(\mathcal{A}_{3}\right)=-1, \\
& \chi\left(B_{1}\right)=+1, \quad \chi\left(B_{2}\right)=+1, \quad \chi\left(B_{3}\right)=+1 .
\end{aligned}
$$

By the usual theory of covering surfaces $[2,29], \chi$ determines a two-sheeted covering $F$ of $F$ having genus $\tilde{g}=5$. The following three figures illustrate the situation.

In figure 7, $\left\{\alpha_{k}, \beta_{k}\right\}_{k=1}^{3}$ is a canonical homology basis. Figure 8 shows the 2-sheeted covering $\tilde{F}$. And, in figure 9, we readily check that $\left\{\bar{\alpha}_{k}, \bar{\beta}_{k}\right\}_{k=1}^{5}$ is a canonical homology basis for $\tilde{F}$. (The $\bar{\alpha}_{k}, \vec{\beta}_{k}$ are non-dividing cycles with the correct intersection numbers.) Recall [2, p. 71] and [60, p. 123].

We want to calculate $m_{\tilde{F}}[z]$. To do so, we shall deform $\left\{\bar{\alpha}_{k}, \bar{\beta}_{k}\right\}_{k=1}^{5}$ into a canonical dissection $\left\{\vec{A}_{k}, \bar{B}_{k}\right\}_{k=1}^{5}$ using the paths $c_{j}$ mentioned above. Then, using a minor abbreviation,

$$
m_{\tilde{F}}[z]=\left[\tilde{\mathcal{A}_{1}}, \ldots, \tilde{\mathcal{A}}_{5} ; \tilde{B}_{1}, \ldots, \tilde{\mathcal{B}}_{5}\right] .
$$

Since the curves $\bar{\alpha}_{k}, \bar{A}_{k}$ and $\bar{\beta}_{k}, \bar{B}_{k}$ are freely homotopic, we obtain

$$
M\left[\xi\left(\overline{\mathcal{A}}_{k}\right)\right]=M\left[\xi\left(\bar{\alpha}_{k}\right)\right], \quad M\left[\xi\left(\overline{\mathcal{B}}_{k}\right)\right]=M\left[\xi\left(\bar{\beta}_{k}\right)\right] .
$$

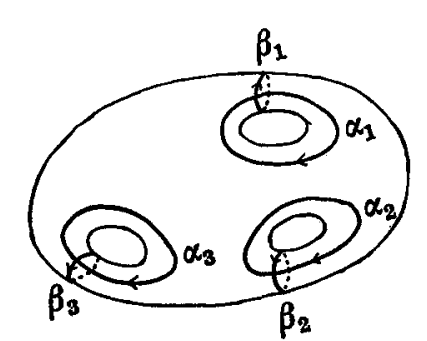

Fig. 7

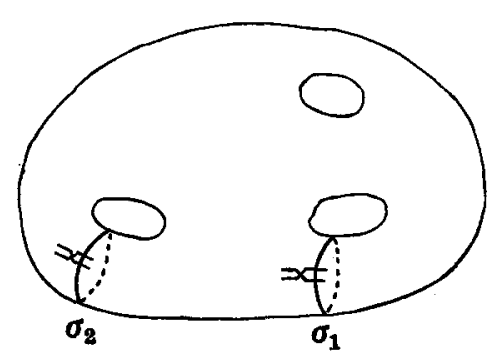

Fig. 8. $\sigma_{2}$ and $\sigma_{2}$ are the branch lines. 


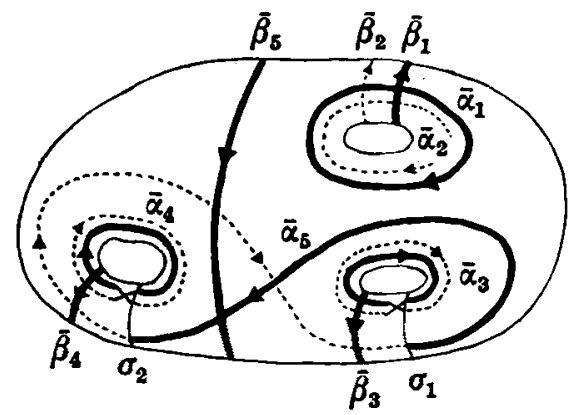

Fig. 9. $\sigma_{1}$ and $\sigma_{2}$ are the branch lines. _ , Top sheet; - - , bottom sheet. The "back side" is

By inspection of figures 7-9, we deduce that

$$
\begin{aligned}
& M\left[\xi\left(\bar{\alpha}_{1}\right)\right]=M\left[\mathcal{A}_{1}\right] ; \quad M\left[\xi\left(\bar{\alpha}_{2}\right)\right]=M\left[\mathcal{A}_{1}\right] ; \\
& M\left[\xi\left(\bar{\alpha}_{3}\right)\right]=M\left[\mathcal{A}_{2}^{2}\right] ; M\left[\xi\left(\bar{\alpha}_{4}\right)\right]=M\left[\mathcal{A}_{3}^{2}\right] ; \\
& M\left[\xi\left(\bar{\alpha}_{5}\right)\right]=M\left[\mathcal{A}_{3} \mathcal{A}_{2}^{-1}\right] ; \\
& M\left[\xi\left(\bar{\beta}_{1}\right)\right]=M\left[B_{1}\right] ; \quad M\left[\xi\left(\bar{\beta}_{3}\right)\right]=M\left[B_{1}\right] ; \\
& M\left[\xi\left(\bar{\beta}_{3}\right)\right]=M\left[B_{2}\right] ; \quad M\left[\xi\left(\bar{\beta}_{4}\right)\right]=M\left[B_{3}\right] ; \\
& M\left[\xi\left(\bar{\beta}_{5}\right)\right]=M\left[\left(\mathcal{A}_{3}, \mathcal{B}_{3}\right)\right] .
\end{aligned}
$$

These computations are all made by noting the appropriate free homotopies.

Using the definition of $z$, we now obtain:

$$
\begin{gathered}
M\left[\xi\left(\bar{\alpha}_{1}\right)\right]=T_{1} ; \quad M\left[\xi\left(\bar{\alpha}_{2}\right)\right]=T_{1} ; \\
M\left[\xi\left(\bar{\alpha}_{3}\right)\right]=T_{2}^{2}=I ; \quad M\left[\xi\left(\bar{\alpha}_{4}\right)\right]=T_{3}^{2}=I \quad \bmod m_{F}[z] ; \\
M\left[\xi\left(\bar{\alpha}_{5}\right)\right]=T_{3} T_{2}^{-1}=I ; \\
M\left[\xi\left(\bar{\beta}_{1}\right)\right]=I ; \quad M\left[\xi\left(\bar{\beta}_{2}\right)\right]=I ; \\
M\left[\xi\left(\bar{\beta}_{3}\right)\right]=I ; \quad M\left[\xi\left(\bar{\beta}_{4}\right)\right]=I \quad \bmod m_{F}[z] ; \\
M\left[\xi\left(\bar{\beta}_{5}\right)\right]=I .
\end{gathered}
$$

It follows at once that

$$
m_{\tilde{F}}[z]=\left[W_{1}, W_{2}, I, I, I ; I, I, I, I, I\right]
$$

where $W_{1} \equiv T_{1} \equiv W_{2} \bmod m_{F}[z]$.

In order to state the corresponding theorem, we let $E_{0}$ be the set of all $\tau \in T_{\sigma}$ which support locally schlicht L.P. functions $z$ having $m[z]$ of the form $\left[W_{1}, W_{2}, I, \ldots, I\right]$, that is, with $2 g-2 I$ entries. 
THसОR-M 13. The exceptional set $E_{g}$ has measure zero in $T_{g}$ provided that $g \geqslant 3$. In general, however, $E_{0}$ is non-empty.

Proof. We have just seen that $E_{5}$ is non-empty. A similar (though easier) construction works for $E_{3}$. One expects, of course, that all $E_{g}, g \geqslant 3$, are nonvoid.

To prove that $E_{g}$ has measure zero in $T_{g}$, we simply lift the locus $\left[W_{1}, W_{2}, I, \ldots, I\right]$ $\bmod L F$ up to $T Q$ by using the local diffeomorphism $p: T Q \rightarrow m$ countably many times. The corresponding locus in $T Q$ is therefore a countable union of local pieces, each having complex dimension $\leqslant 3$. Since $3<3 g-3$, an application of the obvious projection $T Q \rightarrow T_{\sigma}$ shows that $E_{g}$ has measure zero.

Remark. Needless to say, $m[z]=\left[W_{1}, I, \ldots, I\right]$ is impossible by Theorem B.

The last result we want to discuss here concerns the Nevanlinna characteristic function $T(r)$ of a locally schlicht L.P. function $z(t)$. This sort of Nevanlinna theory can be found, for example, in [64, chapter 11] in the context of Fuchsian groups. We recall that

$$
T(r)=\frac{1}{\pi} \int_{r_{0}}^{r} \frac{A(x)}{x} d x,
$$

where $A(x)$ is the spherical area of the Riemannian image $z\{|t| \leqslant x\}$.

THEOREM 14. Let the previous notation apply. There are then exactly two possibilities:

(a) if $z(U) \neq \hat{\mathrm{C}}$, then $T(r)$ is bounded;

(b) if $z(U)=\hat{\mathbf{C}}$, then the order of growth of $T(r)$ is precisely $\ln 1 /(1-r)$.

Proof. To handle case (a), we use Theorem D. The action of $m[z]$ on $z(U)$ is thus properly discontinuous. Using theorems like $B$ and $C$, we readily check that the complement $E$ of $z(U)$ contains at least three points. See also [5, pp. 305-307], [36, p. 542]. The Kleinian group $m[z]$ is therefore non-elementary, so a well-known result of P. J. Myrberg [40] guarantees that Cap $(E)>0$. The classical Nevanlinna-Frostman theorem [41, p. 272] then shows that $T(r)$ is bounded.

In case (b), we proceed as follows. Let $₹$ be the customary fundamental polygon for the automorphic group. Set: $P_{1}(r)=$ the number of polygons $L(\mathcal{F})$ contained entirely within $\{|t| \leqslant r\} ; P_{2}(r)=$ the number of polygons $L(\mathcal{F})$ which have nonvoid intersection with $\{|t| \leqslant r\}$. Since $\mathcal{F}$ is compact, elementary considerations show that

$$
\frac{c_{1}}{1-r} \leqslant P_{1}(r) \leqslant P_{2}(r) \leqslant \frac{c_{2}}{1-r}, r \rightarrow 1
$$

A similar estimate clearly holds when $\mathfrak{F}$ is replaced by a finite union $\mathcal{D}=L_{1}(\mathfrak{F}) \cup \ldots \cup L_{N}(\mathfrak{F})$. 
Using the upper bound on $P_{2}(r)$, and the fact that $z(t)$ is a locally schlicht L.P. function, we easily see that $T(r) \leqslant c_{3} \ln 1 /(1-r)$. To get a lower bound on $T(r)$, simply choose $D$ so that $z(\mathcal{D})=\hat{\mathbf{C}}$. (This is easily seen to be possible.) The lower bound on $P_{1}(r)$ can then be used to show that $T(r) \geqslant c_{4} \ln 1 /(1-r)$.

Corresponding results for the second main theorem (in Nevanlinna theory) would be of definite interest $[41$, p. 272], $[64$, p. 554].

\section{Linearly polymorphic functions with ramification}

In this next to the last section, we would like to indicate very briefly how one can obtain information about locally schlicht L.P. functions by studying, instead, L.P. functions with points of ramification (critical points).

The results we obtain are all based on a certain geometric method whose main ideas seem sufficiently rigorous to be presented here. The method itself has not yet been fully developed, so that a more detailed exposition must (unfortunately) be postponed. As will be seen, the method is quite widely applicable and fits in very naturally among the techniques of section 9.

The first step is to develop two results, Theorems 15 and 16 , which will serve as substitutes for Theorems $A$ and 2, respectively.

We let $\tau \in T_{q}$ be any marked compact Riemann surface of genus $g \geqslant 2$ and let $(U, \pi)$ be its universal covering. Recall that an L.P. function is, by definition, simply a meromorphic function $z(t)$ on $(U, \pi)$ which transforms linear fractionally $z(L t)=\mathscr{L} z(t)$ under the automorphic group $\mathcal{G}$. The marked monodromy group $m[z]$ can then be defined in the usual way. We observe that the Riemannian image $z(\mathcal{F})$ will be a simply-connected membrane, except that there may be points of ramification. We shall let $\theta[z]=$ the total ramification of the membrane $z$ (F).

One naturally tries to extend the previous developments to the present general case (ramified or not). For example, let $R_{k}$ be the conglomeration of all marked monodromy groups $m[z]$ with $\theta[z]=k$. Similarly, let $R_{k}(\tau)$ arise from those $z$ on a fixed surface $\tau \in T_{\mathbf{v}}$. It would be of great interest to completely describe $R_{k}$ and $R_{k}(\tau)$. (One should also recall here Lemma 1 of section 4.)

We remark that the spaces $R_{k}$ for $0 \leqslant k<2 g-2$ are definitely proper subsets of $N$, since Theorem B still applies. This fact can be seen by a simple adaptation of [5, pp. 297300]: if $M[w]$ contains only linear maps $a z+b$, then the divisor of the (local) differential $d w$ must have degree $2 g-2$. 
We should also note that by taking $n \rightarrow \infty$ in $\bigcup_{k=0}^{n} R_{k}(\tau)$, one is led to the classical Riemann problem cited in the introduction (section 1 ).

After these preliminary remarks, we can now state the analogues of Theorems A and 2.

THEOREM 15. Let $z=z(t)$ and $w=w(t)$ be L.P. functions on the same surface $\tau \in T_{g}$. Assume that $m[z]=m[w]$ and $\theta[z]+\theta[w]<4 g-4$. Then $z(t) \equiv w(t)$.

THEOREM 16. Let $z=z(t), w=w(s)$ be two L.P. functions on the respective surfaces $\tau_{t}, \tau_{s}$ in $T_{o}$. Assume that $m[z]=m[w]$ and $\theta[z]+\theta[w] \neq 4 g-4$. Then $z(U) \cap w(U)$ is non-empty.

Proof (Theorem 15). Suppose that $z(t) \neq w(t)$. As in the proof of Theorem 2, define

$$
E(t)=\frac{[w(t)-z(t)]^{2}}{w^{\prime}(t) z^{\prime}(t)}, t \in U
$$

We easily check that $E(L t)=E(t) L^{\prime}(t)^{2}$ for $L \in \mathcal{G}$ and that $E(t)$ is left unchanged under an auxiliary L.F. mapping $(z, w) \rightarrow(M z, M w)$. To study the local behavior of $E(t)$, it will suffice to examine two cases: (a) $z(t)=t^{m}+O\left(t^{m+1}\right), w(t)=c t^{n}+O\left(t^{n+1}\right)$; (b) $z(t)=t^{m}+O\left(t^{m+1}\right)$, $w(t)=1+c t^{n}+O\left(t^{n+1}\right)$. Here $c \neq 0$ and $n \geqslant m \geqslant 1$. Case (a) gives $E(t) \sim c_{1} t^{2 m+\delta} t^{2-m-n}$ with $\delta \geqslant 0$, while case (b) gives $E(t) \sim c_{2} t^{2-m_{-n}}$. The quadratic differential $E(t)^{-1} d t^{2}$ must therefore have

$$
\theta[z]+\theta[w]-\sum_{(a)}[2 m+\delta]=4 g-4
$$

This yields a contradiction.

Proof (Theorem 16). This proof will be based on that of Theorem 2. We introduce the usual orientation-preserving diffeomorphisms $\eta=f(\xi), s=\phi(t)$ to connect $\tau_{t}$ and $\tau_{s}$, and assume that the ramification points of $z(t)$ and $w[\phi(t)]$ are contained in the set $\left\{t_{1}, \ldots, t_{N}\right\}$ $\bmod \mathcal{G}_{t}\left(N\right.$ even). Introduce points $s_{i}, \xi_{i}, \eta_{i}$ and multiplicities $m_{i}, n_{i}$ in the obvious way:

$$
\theta[z]=\sum_{i=1}^{N}\left(m_{i}-1\right), \theta[w]=\sum_{i=1}^{N}\left(n_{i}-1\right) .
$$

Using the diffeomorphism $f$, we now form 2-sheeted ramified coverings of $\tau_{t}$ and $\tau_{s}$ with branch points $\xi_{1}, \ldots, \xi_{N}$ and $\eta_{1}, \ldots, \eta_{N}$. These coverings are constructed so as to have branch lines $\xi_{2 k-1} \xi_{2 k}, \eta_{2 k-1} \eta_{2 k}$ for $1 \leqslant k \leqslant N / 2$. In this way, we obtain marked surfaces $\tilde{\tau}_{t}$ and $\tilde{\tau}_{s}$ connected by the obvious homeomorphism $f$. The main point here is that the L.P. functions $z$ and $w$ lift to $\tilde{\tau}_{t}$ and $\tilde{\tau}_{s}$ in an obvious fashion. The resulting L.P. functions $\tilde{z}$ and 
$\tilde{w}$ are then locally schlicht except at the branch points, where their respective multiplicities are $2 m_{i}, 2 n_{i}$.

We readily check that: (i) the genus of $\tilde{\tau}_{t}$ is $G=2 g-1+\frac{1}{2} N$ [41, p. 324]; (ii) $\theta[\tilde{z}]=$ $2 \theta[z]+N, \theta[\tilde{w}]=2 \theta[w]+N$; (iii) $\theta[\tilde{z}]+\theta[\tilde{w}] \neq 4 G-4$. A moment's thought shows that $m[\tilde{z}]=m[\tilde{w}], \tilde{z}(U) \cap \tilde{w}(U)=z(U) \cap w(U)$. Hence, WLOG, we may assume from the very start that $m_{i}+n_{i} \equiv 0(\bmod 2)$.

Suppose that $z(U) \cap w(U)$ were empty. We then form $E(t)$ as in the proof of Theorem 2 and must clearly study the behavior of $E(t)$ at the (possible) critical points $t_{1}, \ldots, t_{N}$. We quickly see that $E(t)$ behaves like $\left(t-t_{i}\right)^{1-m_{i}}\left[\phi(t)-\phi\left(t_{i}\right)\right]^{1-n_{i}}$. Since $s=\phi(t)$ is an orientationpreserving diffeomorphism and $m_{i}+n_{i} \equiv 0(\bmod 2)$, it is easily checked that $D(t)=\sqrt{E(t)}$ is well-defined for $t \neq t_{i} \bmod \mathcal{G}_{t}$.

Therefore, as in Theorem 2, $D(L t)=\chi(L) D(t) L^{\prime}(t)$ for a unique homomorphism $\chi$ with $\chi(L)= \pm 1$, and so $D(t)$ defines a vector field with singularities on the appropriate unramified 2-sheeted covering $\tau_{t}(\chi)$ of $\tau_{t}$. An application of the Hopf-Poincaré index theorem forces us to conclude that $\theta[z]+\theta[w]=4 g-4$. This is a contradiction.

Remark 1. Equation (11.1) clearly shows that Theorem 15 can be improved somewhat. Nevertheless, the example $w(t)=z(t)+C$, where $C \neq 0$ and $z(t)$ is an Abelian integral of the first kind, should be noted.

Remark 2. Theorem 15 and a previous remark (about $a z+b$ ) show that $R_{k} \subseteq N_{1}$ for $0 \leqslant k<2 g-2$. See also $[5$, pp. 55-64].

Remark 3. For further information about $R_{k}(\tau)$ and Theorem 15, one may wish to refer to [38]. Cf. also [87].

Remark 4. The remark following the proof of Theorem 2 is applicable to Theorem 16 as well.

We can now turn to the second stage of the investigation. As we have seen in section 8 , the space $R_{0}$ contains groups arbitrarily close to the identity group $[I, \ldots, I]$. Motivated by [5, pp. 337-341], we ask whether there can exist spaces $R_{0}(\tau)$ with the same property. There are of course similar questions for any of the excluded cases given in section 7 .

Using the spaces $R_{k}(\tau)$ and Theorem 15, we are able to provide evidence in support of the following conjectures:

(A) $R_{0}(\tau)$ is bounded away from any given translation group $\left[U_{1}, \ldots, V_{\theta}\right]$ for every $\tau \in T_{g}$

$\left(\mathrm{A}_{\mathrm{T}}\right) R_{0}(\tau)$ is bounded away from $[I, \ldots, I]$ for every $\tau \in T_{g}$. A translation group $\left[U_{1}, \ldots, U_{g} ; V_{1}, \ldots, V_{\ell}\right]=[U ; V]$ is simply one in which the $U_{k}, V_{k}$ are 
all translations. As will be seen, our evidence for $\left(A_{I}\right)$ is much more convincing than that for (A).

The method we use to get these results is probably of greater interest than the conjectures themselves. Its underlying idea is quite simple. Suppose that $w$ is an L.P. function on $\tau_{0} \in T_{g}$ with $k$ simple branch points, $3 g \leqslant k<4 g-4$. Choose any group [ $\left.U ; V\right] \in N$ close to $m[w]$. We can then deform the fundamental membrane $w(\mathfrak{F})$ slightly to obtain a membrane $\mathcal{D}$ having $[U ; V]$ identifications. (We recall here (9.1)-(9.5).) The membrane $\mathcal{D}$ will clearly have $k$ simple branch points, but will not in general correspond to $\tau_{0} \in T_{q}$. However, by a slight perturbation of exactly $3 g-3$ of the branch points, one can presumably obtain a $[U ; V]$ membrane which does correspond to $\tau_{0}$; see, for example, [17] and [49]. The fixed branch points serve to rule out auxiliary L.F. mappings. An application of Theorem 15 now shows that $[U ; V] \notin R_{0}(\tau)$.

Due to limitations of space, we shall proceed somewhat informally. We begin with case $\left(\mathrm{A}_{\mathrm{I}}\right)$. A general point $\tau \in T_{g}$ can be represented as a marked surface $F=F\left(a_{1}, \ldots, a_{w}\right)$ over the sphere $\hat{\mathbf{C}}$ so that: (a) $F$ has sheet number $n=[(g+3) / 2]$; (b) the branch points $a_{1}, \ldots, a_{w}$ of $F$ are all simple and have distinct projections; $(\mathrm{c})\left(a_{1}, \ldots, a_{w}\right) \in \mathbf{C}^{w},\left(a_{w-2}, a_{w-1}, a_{w}\right)=$ $(0,1,2)$. This can be seen intuitively using the Riemann-Hurwitz formula $w=2 n+2 g-2$ :

$$
w=\left\{\begin{array}{ll}
3 g, & g \text { even } \\
3 g+1, & g \text { odd }
\end{array} .\right.
$$

See also $[14],[26$, p. 532], [59, p. 380].

For normalized points $\left(b_{1}, \ldots, b_{w}\right) \approx\left(a_{1}, \ldots, a_{w}\right)$, we may define the marked surfaces $F\left(b_{1}, \ldots, b_{w}\right)$ in an obvious way. The surfaces $F(b)$ can all be given the same canonical dissection. We let $f_{b}: F(b) \rightarrow \hat{\mathbf{C}}$ be the obvious projection. Now, it is very plausible that the mapping

$$
\Phi:\left(b_{1}, \ldots, b_{w}\right) \rightarrow F\left(b_{1}, \ldots, b_{w}\right) \rightarrow T_{g}
$$

is locally biholomorphic provided that: (i) $b_{w-j}=a_{w-j}, 0 \leqslant j \leqslant 2, g$ even; (ii) $b_{w-j}=a_{w-j}$, $0 \leqslant j \leqslant 3, g$ odd. We shall therefore assume (i) and (ii) to exclude auxiliary L.F. mappings. Note that we are using here the fact that $T_{g}$ is a complex analytic manifold [8, 13].

Consider points $[U ; V] \in N$ near $[I ; I]$ and points $b \approx a$. We form the simply-connected membrane associated with the L.P. function $f_{b}$ and the fixed canonical dissection of $F(b)$. This membrane has $[I ; I]$ identifications and branch points $b_{1}, \ldots, b_{w}$. We readily distort the membrane near its boundary to obtain a new membrane with the same branch points for $[U ; V]$. Let the corresponding Teichmüller point be $T(b ; U ; V)$. Define

$$
c(b ; U ; V)=\Phi^{-1}[T(b ; U ; V)] .
$$


The mapping $c(b ; U ; V)$ is essentially a mapping $\mathbf{C}^{30-3} \rightarrow \mathbf{C}^{30-3}$ for each $(U ; V)$. Also observe that $c(b ; I ; I)=b$.

We would certainly expect that $c(b ; U ; V)$ is complex-analytic in $b$ and continuous (say) in $(U ; V)$. The $b$-partials of $c(b ; U ; V)$ near $a$ can be calculated using the Cauchy integral formula. For that reason, we then have

$$
\frac{1}{2}\left\|b^{\prime}-b^{\prime \prime}\right\| \leqslant\left\|c\left(b^{\prime} ; U ; V\right)-c\left(b^{\prime \prime} ; U ; V\right)\right\| \leqslant 2\left\|b^{\prime}-b^{\prime \prime}\right\|
$$

locally. From this, we immediately deduce the local solvability of $c(b ; U ; V)=a$ for all $[U ; V]$ close enough to $[I ; I]$.

Theorem 15 will now show that $[U ; V] \notin R_{0}(\tau)$ provided that $w<4 g-4$. This will certainly be the case for $g \geqslant 6$.

This is our evidence for the truth of $\left(\mathrm{A}_{\mathrm{I}}\right)$ when $g \geqslant 6$. Of course, if $\left(\mathrm{A}_{\mathrm{I}}\right)$ holds for $g \geqslant 6$, then it also holds for $2 \leqslant g \leqslant 5$, as can be seen by passing to an appropriate $N$-sheeted covering of $\tau$.

We now turn to conjecture (A). Fix $\tau \in T_{g}$ and consider the Abelian integral

$$
u=c_{0}+c_{1} u_{1}+\ldots+c_{g} u_{g}+d_{1} Y_{\xi_{1}}+\ldots+d_{m} Y_{\xi_{m}}, \quad d_{1} \cdots d_{m} \neq 0 .
$$

The $u_{a}$ and $Y_{\xi}$ are normalized Abelian integrals of the first and second kinds [42, pp. 392398]. In the general case, one would expect that $u$ has $m$ simple poles and $2 m+2 g-2$ simple, non-overlapping branch points. The monodromy group of $u$ is a translation group $\left[U_{0} ; V_{0}\right]$.

The fundamental membrane $u(\mathcal{F})$ will have branch points $a_{1}, \ldots, a_{2 m+2 g-2}$. Consider $[U ; V] \in N$ near $\left[U_{0} ; V_{0}\right]$ and points $b \approx a$. By distorting $u(\partial \mathcal{F})$ slightly and pushing $a$ to $b$, we obtain a new membrane $\mathcal{D}(b ; U ; V)$. We obviously want to normalize things so that the mapping

$$
\Phi:(b) \rightarrow \mathcal{D}\left(b ; U_{0} ; V_{0}\right) \rightarrow T_{g}
$$

is locally biholomorphic.

To do so, we may assume that $\left[U_{0} ; V_{0}\right] \neq[I ; I]$ since the case $\left(\mathrm{A}_{\mathrm{I}}\right)$ has already been studied. It follows that $T\left[U_{0} ; V_{0}\right] T^{-1}=\left[U_{0} ; V_{0}\right]$ with $T \in L F(2, \mathrm{C})$ iff $T$ is a translation. For this reason, one may expect that $\Phi$ will be locally biholomorphic provided that $2 g+2 m-2 \geqslant 3 g-2$ and $b_{j}=a_{j}$ for $j \geqslant 3 g-2$. (We need only rule out auxiliary translations this time.) We therefore assume that $m \geqslant \frac{1}{2} g$.

The argument now proceeds as before. Let $T(b ; U ; V)$ be the Teichmüller point corresponding to $\mathcal{D}(b ; U ; V)$ and define

$$
c(b ; U ; V)=\Phi^{-1}[T(b ; U ; V)] .
$$

We may expect that $c(b ; U ; V)$ is complex-analytic in $b$ and continuous in $[U ; V]$. As in 
case $\left(\mathrm{A}_{\mathrm{I}}\right)$, it follows that the equation $c(b ; U ; V)=a$ is solvable whenever $[U ; V]$ is close enough to $\left[U_{0} ; V_{0}\right]$. Theorem 15 implies that $[U ; V] \notin R_{0}(\tau)$ provided that $2 m+2 g-2<$ $4 g-4$, i.e. for $m<g-1$.

It remains to determine which translation groups $\left[U_{0} ; V_{0}\right]$ can be realized in this way under the restriction

$$
\frac{1}{2} g \leqslant m<g-1 .
$$

In the notation of $[42, \mathrm{pp} .360,366,397]$ the Abelian integral $u$ has periods:

$$
\begin{aligned}
& U_{k}(z)=z+c_{k} ; \\
& V_{k}(z)=z+\sum_{j=1}^{g} c_{j} \tau_{j k}-2 \pi i \sum_{j=1}^{m} d_{j} \Phi_{k}\left(\xi_{j}\right) .
\end{aligned}
$$

Suppose that $\tau \in T_{g}$ is general. We can then take $\tau=F\left(a_{1}, \ldots, a_{w}\right)$ as in case $\left(\mathrm{A}_{\mathrm{I}}\right)$ and let $f: F(a) \rightarrow \hat{\mathbf{C}}$ be the usual function. Therefore

$$
f=c_{0}+d_{1} Y_{\xi_{1}}(t)+\ldots+d_{n} Y_{\xi_{n}}(t),
$$

with $n=[(g+3) / 2], d_{1} \cdots d_{n} \neq 0$, and $\left\{\xi_{1}, \ldots, \xi_{n}\right\}$ distinct $\bmod \mathcal{G}$. Since $f$ is single-valued, the period relations yield

$$
\sum_{j=1}^{n} d_{j} \Phi_{\alpha}\left(\xi_{j}\right)=0,1 \leqslant \alpha \leqslant g .
$$

We shall consider points $(x ; \eta) \in \mathbf{C}^{2 n}$ near $(d ; \xi)$ such that $\left(x_{n} ; \eta_{n}\right)=\left(d_{n} ; \xi_{n}\right)$ for $g$ even, and $\left(x_{n-1}, x_{n} ; \eta_{n}\right)=\left(d_{n-1}, d_{n} ; \xi_{n}\right)$ for $g$ odd. In each case, the number of free variables is $g$.

The corresponding local analytic mapping

$$
\Omega:(x ; \eta) \rightarrow\left(x_{1} \Phi_{\alpha}\left(\eta_{1}\right)+\ldots+x_{n} \Phi_{\alpha}\left(\eta_{n}\right)\right)_{\alpha=1}^{q}
$$

is of interest. Suppose, for example, that $\Omega(x ; \eta)=(0)$. The function $f_{1}=c_{0}+x_{1} Y_{\eta_{1}}(t)+\ldots+$ $x_{n} Y_{\eta_{n}}(t)$ will then yield an $n$-sheeted representation of $\tau$ which approximates that of $f$. Since $\tau$ is general and $f_{1} \approx f$, we conclude that $f_{1}=T f$ for $T \in L F(2, \mathrm{C})[26$, p. 552], [59, p. 380]. By the $(x ; \eta)$ normalization, we deduce that $T(\infty)=\infty$, and then that $T$ is simply a translation. Hence $(x ; \eta)=(d ; \xi)$.

According to a general theorem of Osgood [42, p. 139], one should now expect that $\Omega$ maps onto a complete neighborhood of $(0)$. Incidentally, it is an elementary exercise to check that the Jacobian of $\Omega$ does not vanish identically [42, p. 417]. The system $x_{1} \Phi_{\alpha}\left(\eta_{1}\right)+\ldots+x_{n} \Phi_{\alpha}\left(\eta_{n}\right)=y_{\alpha}(1 \leqslant \alpha \leqslant g)$ will therefore be solvable with $x_{1} \cdots x_{n} \neq 0$ and $\eta \approx \xi$ for any $y \in \mathbb{C}^{g}$. Using (11.3) and the fact that $\operatorname{det}\left(\tau_{j k}\right) \neq 0$, we now see that $c_{1} u_{1}+\ldots+c_{g} u_{g}+x_{1} Y_{\eta_{1}}(t)+\ldots+x_{n} Y_{\eta_{n}}(t)$ can have an arbitrary set of periods [ $\left.U_{0} ; V_{0}\right]$.

For non-exceptional groups $\left[U_{0} ; V_{0}\right]$, the corresponding function $\Sigma c_{k} u_{k}+\Sigma x_{i \hbar} Y_{\eta_{k}}$ will certainly have $n$ simple poles and $2 n+2 g-2$ simple, non-overlapping branch points. 4-752905 Acta mathematica 135. Imprimé le 19 Décembre 1975 
The previous development will then apply with $m=n$, provided that

$$
\frac{1}{2} g \leqslant\left[\frac{g+3}{2}\right]<g-1 \text {, }
$$

that is, for $g \geqslant 6$.

This completes our evidence in support of conjecture (A) for $g \geqslant 6$. The case $2 \leqslant g \leqslant 5$ is again handled by means of an appropriate $N$-sheeted covering of $\tau$.

Remark. The techniques used above can obviously be generalized quite a bit. The general method is, however, amply illustrated by means of the two cases $(A),\left(A_{I}\right)$ that we have considered.

\section{Concluding remarks}

We shall close with a partial list of open problems and directions for further research.

(1) As mentioned in sections 1 and 6, our main goal has been to obtain a better understanding of the mapping $p: T Q \rightarrow m$ and of the subdomain $m \subseteq N_{1} / L F$. Most of our success has been with the former. In this regard, we naturally ask for a better description of the obstructions to the path-lifting property for $p$. It seems likely that such obstructions reflect algebraic degeneracies in the monodromy groups. For example, in our proof of Theorem 8, there was a cusp (so-to-speak).

(2) Thus far we have obtained very little insight into the nature of the spaces $m \subseteq$ $N_{1} / L F \subseteq N / L F$ and $R \subseteq N_{1} \subseteq N$. As mentioned after Theorem 10, Professor Schiffer has suggested that $m$ and $R$ may well be dense. If that be the case, it would be very interesting to describe their complements. See also the remarks following Theorem 10 (proof).

(3) Further study of the submanifolds $m(\tau)$ and $R(\tau)$, associated with L.P. functions on a fixed surface $\tau \in T_{g}$, would definitely be useful. Ideally one should be able to say what $m(\tau)$ and $R(\tau)$ actually look like. There are already certain indications in section 11 . We ask, for example, whether the submanifolds $m(\tau) \subseteq N_{1} / L F, R(\tau) \subseteq N_{1}$ are closed. And: whether the mapping $Q[\tau] \rightarrow\left(\tilde{A}, \ldots, \tilde{A}_{\theta}\right) \bmod L F$ is locally $1-1$. We recall here (6.8), (6.11), and Theorem 12.

(4) It seems clear that the methods of section 11 can be carried much further. Questions for $m, R, m(\tau), R(\tau)$ have obvious analogues for $m_{k}, R_{k}, m_{k}(\tau), R_{k}(\tau)$. We might point out that by "attaching" surfaces like $w^{n}=z^{n-1}(z-1)$ to the fundamental membranes, one can prove that $R_{k} \subseteq R_{k+2 m}$ for $m \geqslant 1$.

(5) What does $R_{k}(\tau)$ look like? A geometric argument (as in section 11) or an analytic one (based on Lemma 1) seems to show that $\operatorname{dim} R_{k}(\tau)=\min [3 g+k, 6 g-3]$. There are obvious connections here with the classical Riemann problem (i. e.when $\left.R_{i}(\tau)=N\right)$. 
(6) To what extent can Nevanlinna theory be used to describe the mapping properties of L.P. functions? Recall Theorem 14. See also [21].

(7) As is apparent, we have made no real attempt to develop an existence theory for monodromy groups in this paper. Deeper investigation of this area is definitely called for. See, for example, [47], [48], [51], [55], and [65].

(8) The study of problem $A$ in section 1 along the lines of a variational theory quickly leads one to some fascinating connections with Eichler cohomology, Eichler integrals, and Prym differentials. These relations certainly deserve closer investigation. See [19], [20], [24], [25].

(9) Further study of finite monodromy groups may be interesting. Some references for this are [16, pp. 174-218], [27, pp. 524-529].

(10) Monodromy groups in higher dimensions (i.e. for higher-order D.E.) would certainly appear to deserve further study. See [16, pp. 191-218], [27], [47], [54, pp. 180-199], and [81]. One should also recall the general remarks of section 3.

(11) Finally, for some number-theoretic problems related to zeta-Fuchsian series and monodromy groups, we may refer to [43], [58], and [65].

\section{References}

[1]. Ahlfors, L. \& Bers, L., Riemann's mapping theorem for variable metrics. Ann. of Math., 72 (1960), 385-404.

[2]. Ahlfors, L. \& Sario, L., Riemann Surfaces. Princeton Univ. Press, 1960.

[3]. AhLfors, L., Lectures on Quasi-contormal Mappings. Van Nostrand, 1966.

[4]. Aleksandrov, P. S., Combinatorial Topology, vol. 1. Graylock Press, 1956.

[5]. Appell, P., Gounsat, E. \& Fatou, P., Théorie des Fonctions Algébriques, vol. 2. GauthierVillars, 1930.

[6]. Baker, H. F., Abelian Functions. Cambridge Univ. Press, 1897.

[7]. Bateman, H., Higher Transcendental Functions, vol. 1. McGraw-Hill, 1953.

[8]. Bers, L., Spaces of Riemann surfaces. Proc. Internat. Cong. Math. Edinburgh, (1958), 349-361.

[9]. — Quasi-conformal mappings and Teichmüller's theorem. In Analytic Functions, Princeton Univ. Press, 1960, 89-119.

[10]. - Completeness theorems for Poincaré series. In Proc. Internat. Symp. on Linear Spaces, Jerusalem Academic Press, 1960, 88-100.

[11]. - Holomorphic differentials as functions of moduli. Bull. Amer. Math. Soc., 67 (1961), 206-210.

[12]. - On boundaries of Teichmüller spaces and on Kleinian groups I. Ann. of Math., 91 (1970), 570-600.

[13]. - Uniformization, moduli, and Kleinian groups. Bull. London Math. Soc., 4 (1972), 257-300.

[14]. Brirl, A. \& Noether, M., Utber die algebraischen Functionen und ihre Anwendung in der Geometrie. Math. Ann., 7 (1874), 269-310.

[15]. Ford, L., Automorphic Functions. McGraw-Hill, 1929. 
[16]. Forsyth, A. R., Theory of Differential Equations, vol. 4. Cambridge Univ. Press, 1902.

[17]. Garabedian, P., Distortion of length in conformal mapping. Duke Math. J., 16 (1949), 439-459.

[18]. Gunning, R., Special coordinate coverings of Riemann surfaces. Math. Ann., 170 (1967), 67-86.

[19]. — Lectures on Vector Bundles over Riemann Surfaces. Princeton Univ. Press, 1967.

[20]. - Analytic structures on the space of flat vector bundles over a compact Riemann surface. In Several Complex Variables II, Springer Lecture Notes, 185 (1971), 4762.

[21]. Heins, M., The conformal mapping of Riemann surfaces. In Analytic Functions, Prince. ton Univ. Press, 1960, 137-158.

[22]. Hejhal, D., Monodromy groups and linearly polymorphic functions. In Discontinuous Groups and Riemann Surfaces, Princeton Univ. Press, 1974, 247-261.

[23]. — On Schottky and Teichmüller spaces. Advances in Math., 15 (1975), 133-156.

[24]. - Sur les paramètres accessoires pour l'uniformisation de Schottky. C.R. Acad. Sci. Paris, Ser. A-B., 279 (1974), 695, 713.

[25]. - Variational formulas for linearly polymorphic functions. To appear (1976).

[26]. Hensel, K. \& LandsBerg, G., Theorie der algebraischen Funktionen einer Variabeln. B. G. Teubner, 1902.

[27]. Hrıв, E., Lineare Differentialgleichungen im komplexen Gebiet. In Encyclopädie der Math. Wiss. II B 5, B. G. Teubner, 1921, 471-562.

[28]. Hilbert, D., Utber eine Anwendung der Integralgleichungen auf ein Problem der Funktionentheorie. Verh. Internat. Math. Kongr, Heidelberg, (1904), 233-240.

[29]. Hilton, P. \& Wylie, S., Homology Theory. Cambridge Univ. Press, 1960.

[30]. Kelley, J. L., General Topology. Van Nostrand, 1955.

[31]. KLEIN, F., Vorlesungen über das Ikosaeder. B. G. Teubner, 1884.

[32]. - Ausgewählte Kapitel aus der Theorie der linearen Differentialgleichungen zweiter Ordnung. Göttingen, 1891. (Handwritten notes.)

[33]. — Über lineare Differentialgleichungen der zweiten Ordnung. Göttingen, 1894 (Handwritten notes.)

[34]. — Lectures on Mathematics (The Evanston Colloquium). MacMillan, 1894, especially pp. 33-40.

[35]. — Vorlesungen über die Hypergeometrische Funktion. Springer-Verlag, 1933.

[36]. Kra, I., Deformations of Fuchsian groups. Duke Math. J., 36 (1969), 537-546.

[37]. LaNa, S., Introduction to Transcendental Numbers. Addison-Wesley, 1966.

[38]. Mandelbaum, R., Branched structures and affine and projective bundles on Riemann surfaces. Trans. Amer. Math. Soc., 183 (1973), 37-58.

[39]. Maskit, B., On a class of Kleinian groups. Ann. Acad. Sci. Fenn. Ser A I, 442 (1969), 1-8.

[40]. Myrberg, P. J., Die Kapazität der singulären Menge der linearen Gruppen. Ann. Acad. Sci. Fenn. Ser. A I, 10 (1941) 1-19.

[41]. Nevanlinna, R., Analytic Functions. Springer-Verlag, 1970.

[42]. OsGOod, W. F., Lehrbuch der Funktionentheorie, vol. 2 (two parts). B. G. Teubner, 1929.

[43]. Petersson, H., On a certain kind of zeta-Fuchsian functions. In Report of an International Colloquium on Zeta Functions. Tata Inst. (Bombay), 1956, 297-298.

[44]. Picard, E., Mémoire sur la théorie des fonctions algébriques de deux variables. $J$. Math. Pures Appl., (4 ${ }^{\ominus}$ série), 5 (1889), 135-319.

[45]. PlemelJ, J., Riemannsche Funktionenscharen mit gegebener Monodromiegruppe. Monatshefte für Math. und Physik, 19 (1908), 211-246.

[46]. Poincaré, H., Mémoire sur les fonctions fuchsiennes. Acta Math., 1 (1882), 193-294. 
[47]. — Sur les groupes des équations linéaires. Acta Math., 4 (1884), 201-312.

[48]. - Mémoire sur les fonetions zétafuchsiennes. Acta Math., 5 (1884), 209-278.

[49]. RAUCH, H., Weierstrass points, branch points, and moduli of Riemann surfaces. Comm. Pure Appl. Math., 12 (1959), 543-560.

[50]. Riemann, B., Gesammelte Mathematische Werke, zweite Auflage. B. G. Teubner, 1892.

[51]. RitTer, E., Über Riemann'sche Formenschaaren auf einem beliebigen algebraischen Gebilde. Math. Ann., 47 (1896), 157-221.

[52]. RöHRL, H., Holomorphic fiber bundles over Riemann surfaces. Bull. Amer. Math. Soc., 68 (1962), 125-160.

[53]. Schiffer, M. \& HawLeY, N., Half-order differentials on Riemann surfaces. Acta Math., $115(1966), 199-236$.

[54]. Sohimstnger, L., Handbuch der Theorie der linearen Differentialgleichungen, vol. 2. B. G. Teubner, 1897.

[55]. - - Vorlesungen über lineare Differentialgleichungen. B. G. Teubner, 1908.

[56]. Sсноттку, F., Über die conforme Abbildung mehrfach zusammenhängender ebener Flächen. J. Reine Angew. Math., 83 (1877), 300-351.

[57]. - Uber eine specielle Function, welche bei einer bestimmten linearen Transformation ihres Arguments unverändert bleibt. J. Reine Angew. Math., 101 (1887), 227-272.

[58]. SELBERG, A., Harmonic analysis and discontinuous groups in weakly symmetric Riemannian spaces with applications to Dirichlet series. In Report of an International Colloquium on Zeta Functions, Tata Inst. (Bombay), 1956, 47-87.

[59]. Severr, F., Vorlesungen über Algebraische Geometrie. B. G. Teubner, 1921.

[60]. Sregex, C. L., Topics in Complex Function Theory, vol. 2. Wiley-Interscience, 1971.

[61]. Stemandod, N., The Topology of Fibre Bundles. Princeton Univ. Press, 1951.

[62]. Stoker, J. J., Differential Geometry. Wiley-Interscience, 1969.

[63]. Thichmüller, O., Bestimmung der extremalen quasikonformen Abbildungen bei geschlossenen orientierten Riemannschen Flächen. Abh. Preuss. Akad. Wiss. (Berlin), 4 (1943), 1-42.

[64]. TsuJI, M., Potential Theory in Modern Function Theory, Maruzen, 1959.

[65]. Werl, A., Généralisation des fonctions abéliennes. J. Math. Pures Appl., (9 ${ }^{\circ}$ série), 17 (1938), 47-87.

[66]. Wells, R., Differential Analysis on Complex Manifolds. Prentice-Hall, 1973.

[67]. WirczYnski, E. J., Projective Differential Geometry of Curves and Ruled Surfaces, B. G. Teubner, 1906.

\section{Additional references}

[68]. APPELL, P., Fonctions hypergéométriques et hypersphériques. Gauthier-Villars, 1926.

[69]. Deligne, P., Equations Différentielles a Points Singuliers Réguliers. Springer Lecture Notes, 163 (1970).

[70]. Dewigne, P. \& Katz, N., Groupes de Monodromie en Géométrie Algébrique. Springer Lecture Notes, 340 (1973).

[71]. Erchler, M., Eine Verallgemeinerung der Abelschen Integrale. Math. Z., 67 (1957), 267-298.

[72]. Fricke, R., Automorphe Funktionen mit Einschluss der elliptischen Modulfunktionen. In Encyklopädie der Math. Wiss., II B 4, B. G. Teubner, 1921, 349-470, especially pp. 464-466.

[73]. Fuchs, L., Gesammelte Mathematische Werke. Mayer and Müller, Berlin, 1904-1909, (papers 8, 13, 68, 70). 
[74]. Garnier, R., Solution du problème de Riemann. Ann. Sci. École Norm. Sup., 43 (1926), 177-307.

[75]. — Sur le problème de Plateau. Ann. Sci. École Norm. Sup., 45 (1928), 53-144.

[76]. Griffiths, P., Periods of integrals on algebraic manifolds III. I.H.E.S. Publications, 38 (1970), 125-180. [See also Bull. Amer. Math. Soc., 76 (1970), 228-296.]

[77]. - Complex-analytic properties of certain Zariski open sets on algebraic varieties. Ann. of Math., 94 (1971), 21-51.

[78]. Gunning, R., Some multi-variable problems arising from Riemann surfaces. Actes Congr. Internat. Math. Nice, vol. 2, (1970), 625-626.

[79]. Hejhal, D., Quelques remarques à propos des séries de Poincaré sur les groupes do Schottky. C. R. Acad. Sci. Paris, 280 (1975), 280 (1975), 341-344.

[80]. - Quelques remarques au sujet des fonctions linéairement polymorphes sur une surface de Riemann. C. R. Acad. Sci. Paris, 280 (1975), 637-640.

[81]. - Monodromy groups for higher-order differential equations. Bull. $A M S, 81$ (1975), 590-592.

[82]. HILBERT, D., Grundzüge einer allgemeinen Theorie der linearen Integralgleichungen. B. G. Teubner, 1912, 81-108.

[83]. IHARA, Y., Schwarzian equations. J. Fac. Sci. Univ. Tokyo, 21 (1974), 97-118.

[84]. - On the differentials associated to congruence relations and the Schwarzian equations defining uniformizations. J. Fac. Sci. Univ. Tokyo, 21 (1974), 309-332.

[85]. Kueın, F., Neue Beiträge zur Riemann'schen Functionentheorie. Math. Ann. 21 (1883), 141-218, especially pp. 197-200.

[86]. - Gesammelte Mathematische Abhandlungen, Band 3. Springer Verlag, 1923, pp. 770774 (especially the last paragraph).

[87]. Mandelbaum, R., Unstable bundles and branched structures on Riemann surfaces. Math. Ann., 214 (1975), 49-59.

[88]. Myrberg, P. J., Über die numerische Ausführung der Uniformisierung. Acta Soc. Sci. Fenn., 48 (1920), No. 7, pp. 1-53.

[89]. Picard, E. \& Smart, G., Théorie des Fonctions Algébriques de deux variables indépendantes. Gauthier-Villars, 1897-1906.

[90]. Plemes, J., Utber Schlesingers "Beweis" der Existenz Riemannscher Funktionenscharen mit gegebener Monodromiegruppe. Jber. Deut. Math.-Verein., 18 (1909), 15-20, 340-343.

[91]. — Problems in the sense of Riemann and Klein. Wiley-Interscience, 1964.

[92]. Pochhammer, L., Über hypergeometrischen Funktionen höherer Ordnung. J. Reine Angew. Math., 71 (1870), 316-352.

[93]. Rankin, R. A., Sir Edmund Whittaker's work on automorphic functions. Proc. Edinburgh Math. Soc., 11 (1958), 25-30 (especially the bibliography).

[94]. - The Schwarzian derivative and uniformisation. J. d'Analyse Math., 6 (1958), 149167.

[95]. Schutrrva, F., Beiträge zur geometrischen Theorie der Schwarz'schen $s$-Function. Math. Ann., 44 (1894), 162-260.

[96]. - Die geometrische Theorie der Schwarz'schen $s$-Function für complexe Exponenten. Math. Ann., 46 (1895), 62-76, 529-538.

[97]. Sohlesinger, L., Bemerkungen zum Kontinuitätsbeweise für die Lösbarkeit des Riemanschen Problems. Jber. Deut. Math.*Verein., 18 (1909), 21-25.

[98]. - Bericht ūber die Entwickelung der Theorie der linearen Differentialgleichungen seit 1865. Jber. Deut. Math.-Verein., 18 (1909), 133-266. (This reference contains an extensive bibliography of 1742 items covering the period 1865-1907.)

[99]. Schoenfines, A., Uber Kreisbogenpolygone. Math. Ann., 42 (1893), 377-408. 
[100]. - Utber Kreisbogendreiecke und Kreisbogenvierecke. Math. Ann., 44 (1894), 105-124.

[101]. SchWARz, H. A., Gesammelte Mathematische Abhandlungen, Band 2. Springer Verlag, $1890,211-259,362-368$.

[102]. Thleman, C., Sur les structures homographiques d'une surface de Riemann. Comment. Math. Helv., 33 (1959), 206-211.

[103]. - Sur les structures fibrées osculatrices d'une surface de Riemann. Comm. Math. Helv., 34 (1960), 175-184.

[104]. VANVLECK, E. B., Zur Kettenbruchentwickelung hyperelliptischer und ähnlicher Integrale. Amer. J. Math., 16 (1894), 1-91.

[105]. - On certain differential equations of the second order allied to Hermite's equation Amer. J. Math., 21 (1899), 126-167.

[106]. Vidav, I., Kleinsche Theoreme in der Theorie der linearen Differentialgleichungen. Akad. Znanosti Umetnosti v Liubljani, (1941), 1-63.

[107]. - Les théorèmes de Klein, relatifs aux équations differentielles linéaires à six points singuliers. Slovenska Akad. Znanosti Umetnosti v Ljubljani, (1950), 1-38.

[108]. WILCZYNSKT, E. J., On systems of multiform functions belonging to a group of linear substitutions with uniform coefficients. Amer. J. Math., 21 (1899), 85-106.

[109]. - On linearoid differential equations. Amer. J. Math., 21 (1899), 354-366.

[110]. - On continuous binary linearoid groups, and the corresponding differential equations and $\Lambda$ functions. Amer. J. Math., 22 (1900), 191-225.

[111]. Wirtinger, W., Integrale dritter Gattung und linear polymorphe Funktionen. Monatsh. Math. Phys., 51 (1943), 101-114. (This paper contains some interesting results about theta functions.)

Received October 29, 1974. 